\title{
Composition of modern stream sand derived from a mixture of sedimentary and metamorphic source rocks (Henares River, Central Spain)
}

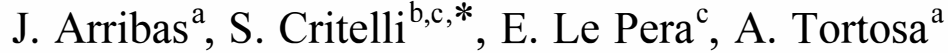 \\ a Departamento de Petrologia y Geoquimica, Facultad de Ciencias Geologicas, Universidad Complutense de Madrid, Madrid, Spain \\ ${ }^{\mathrm{b}}$ Consiglio Nazionale delle Ricerche-Istituto di Ricerca sulle Argille, Via S. Loja-Area Industriale, 85050 Tito Scalo (PZ), Italy \\ ${ }^{\mathrm{c}}$ Consiglio Nazionale delle Ricerche -Istituto di Ricerca per la Protezione Idrogeologica nell'Italia Meridionale ed Insulare, Via Cavour, \\ 87030 Roges di Rende (CS), Italy
}

\begin{abstract}
The Henares River, central Spain, flows westward from the Iberian Range (Mesozoic sedimentary rocks) under semiarid climatic conditions. In the middle and lower reaches, the Henares River receives sediment from three tributaries (Cañamares, Bornova and Sorbe rivers) that drain the Central System (Paleozoic crystalline rocks, low-grade metamorphic rocks, and minor amounts of Mesozoic sedimentary rocks). Modern sands from the Henares River and its tributaries offer an excellent opportunity to evaluate the importance of lithology and physiography in determining detrital modes from mixed metamorphic and sedimentary source terrain. Sand modes from the Henares River and its tributaries are quartzolithic. They plot in a restricted area on a QmFLt diagram, with low contents of feldspar and variable amounts of quartz, and carbonate, metamorphic and minor siliciclastic lithic fragments. Higher feldspar abundances in the Cañamares and Bornova stream sands are related to the erosion of gneissic rocks. By contrast, sands from Sorbe River are low in feldspar, reflecting the absence of coarse metamorphic sources. Thus, proportions of bedrock lithologies in the drainage sub-basins are the main control on detrital modes of the tributaries. In addition, slope acts on the sand productivity of source rocks. Sedimentary source rocks in the upper reaches of the three tributaries have a poor productivity in the carbonate lithic fragments (Lsc), compared with the Lsc productivity of sedimentary sources located downstream, with higher relief. The percentage of bedrock types in the source area versus petrographic indices $(\mathrm{Lm} / \mathrm{L}$, Lss/L and Lsc/L) provide a useful contrast between source and sand composition in the tributaries. Thus, the high content of metamorphic lithic grains in the lithic grain population over-represents this lithology (slate plus schist) at the source terrain. Lithic grains from sedimentary clastic sources generate few recognizable grains (Lss) in the lithic grain population. Local supplies from carbonate sources may produce important increases of Lsc in short reaches of the channels. Sand compositions in the Henares River seem to be very homogeneous in a QmFLt diagram, with only minor differences caused by the supplies from the tributaries. These differences can be modeled in terms of end-member mixing processes between Henares detritus and detritus from the relevant tributary. These mixing processes are more evident when lithic grain contents (LmLvLs and LmLssLsc diagrams) are compared. Changes produced by inputs from the Cañamares, Bornova and Sorbe rivers are not permanent along the Henares course. An important homogenization of
\end{abstract}

\footnotetext{
* Corresponding author.

E-mail addresses: arribas@eucmax.sim.ucm.es (J. Arribas), critelli@irpi.cs.cnr.it (S. Critelli), lepera@irpi.cs.cnr.it (E. Le Pera).
} 
Henares sand composition takes place by the mixing of tributary deposits with previously deposited Henares River terraces. Abrasion during transport does not appear to cause significant changes in the sand composition along the Henares River.

Keywerds: sand; provenance; fluvial basin; central system; Iberian Range; Spain

\section{Introduction}

The provenance of a sediment includes all aspects of the drainage area, including source lithology, topographic relief, climate, transport energy, and hydrodynamics of the sedimentary environment (e.g. Sutmer, 1974; Basu, 1985; Johnsson, 1993). Studies on modern sand contribute to our understanding of the effects of processes controlling the composition of sandsize sediment. This information can be useful for better paleogeographic interpretations of ancient source area/ basin systems (e.g. Dickinson, 1985, 1988; Zuffa, 1987). Such an approach permits the development of actualistic provenance models in sands and their geodynamic implications (e.g. Valloni, 1985; Potter, 1986; DeCelles and Hertel, 1989; Ingersoll et al., 1993), their source lithologies (e.g. Basu, 1976; Mack, 1981; Palomares and Arribas, 1993; Cavazza et al., 1993; Marsaglia, 1993; Critelli and Le Pera, 1999), and physiographic and climatic characteristics of the source area (e.g. Grantham and Velbel, 1988; Johnsson and Stallard, 1989; Ibbeken and Schleyer, 1991; Nesbitt et al., 1996; Critelli et al., 1997; Le Pera and Critelli, 1997; Le Pera, 1998), can be correlated

This study was undertaken to evaluate the relative importance of lithology and physiography in determining the composition of fluvial sands derived from a mixed metamorphic and sedimentary source terrain under semi-arid climatic conditions. The study area is located in central Spain (Fig. 1A), in the interaction zone between the two mountainous systems (the Central System and the Iberian Range) and the corresponding lowland (Southern Castilian Plateau/ Tertiary Tajo Basin). The area was selected because it provides exposures of various metamorphic (Cambrian-Ordovician gneiss, Cambrian-Silurian slates, Ordovician-Silurian metaquartzites, Silurian schists) and sedimentary (clastic rocks, including Permian to Quaternary conglomerates, sandstones, and mudstones and Mesozoic carbonates) source lithologies as well as well-defined drainage basins generating mixed metamorphi-sedimentaclastic sand. The Henares River has the widest of the sampled drainage basins with the headwaters lying in the Iberian Range (Mesozoic sedimentary rocks). In the middle and low courses, the river wanders in the Tajo Basin with a W-SW principal trend, flowing into the Jarama River, which, in turn, debouches into the Tajo River. In these tracts, the Henares River receives supplies from its tributaries (Sorbe, Bornova and Cañamares rivers) draining the Central System (mainly crystalline and metamorphic rocks).

\section{Geological setting and source rock composition}

The Central System Mountains are the southern part of the European Hercynian Chain (Hesperian Massif), and consist of large exposures of granitoids that intrude a metamorphic basement. The Central System experienced intense uplift and erosion following the Alpine Orogeny. Granitoid intrusions are absent in the northeast sector of the chain ('Dominio Oriental' or 'Complejo Somosierra-Ayllon'; e.g. Capote et al., 1982), where exposures of Paleozoic low-rank metamorphic unit prevail. The study area is located in this sector. Here, the oldest metamorphic rocks (Cambrian-Ordovician; Casquet and Fernández-Casals, 1981; Vialette et al., 1986) consist mainly of biotite-bearing orthogneiss $(500-1000 \mathrm{~m}$ in thickess) with feldspar megacrysts, plagioclase, sillimanite, amphibole, garnet, kyanite, staurolite, sphene and epidote (López-Ruiz et al., 1975). The gneissic unit is overlain by an Ordovician to Silurian low-rank metamorphic unit, up to $2600 \mathrm{~m}$ in thickness, represented by an alternation of orthoquartzite and meta-arkose interbedded with black slate-schist Slate-schist includes K-feldspar, plagioclase, biotite, chlorite, chloritoid, sericite, muscovite, apatite, opaques, zircon, tourmaline and garnet (López-Ruiz et al., 1975).

The basement rocks are unconformably overlain by 


\section{A}
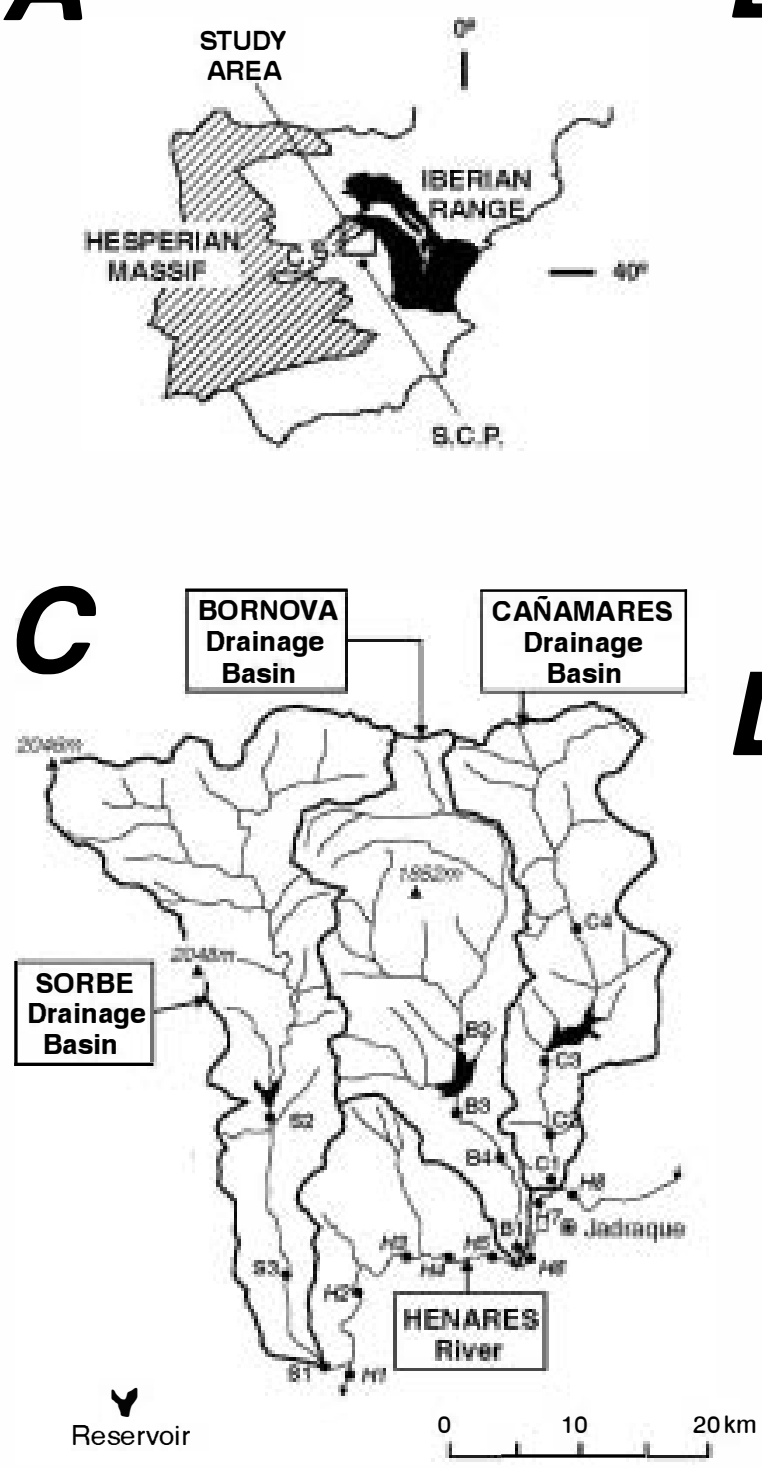
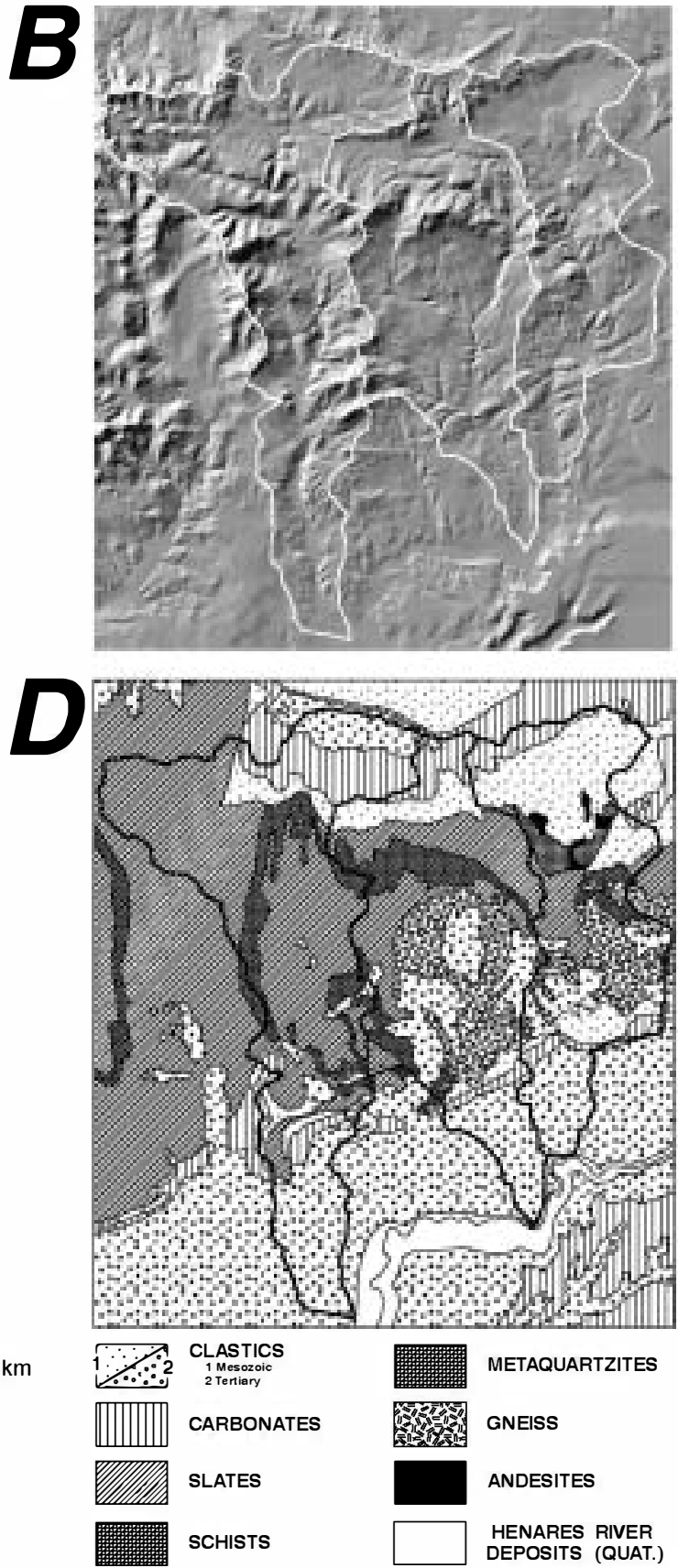

CLASTICS

CARBONATES

SLATES

SCHISTS
MEtAQuARTZITES

TNEISS

ANDESITES

HENARES RIVER DEPOSITS (QUAT)

Fig. 1. Location maps of the study area. (A) Iberian Peninsula showing the distribution of the Hesperian massif and the Iberian Range. C.S., Central System; S.C.P., Southern Castilian Plateau. (B) Digital elevation model (DEM) of the study area. Slopes are emphasized using a northwest illumination obtained by digital filtering. White contours correspond to the drainage basin divides of the Henares River tributaries (Sorbe, Bornova and Cañamares). (C) Drainage network of the Sorbe, Bornova and Cañamares rivers and location of the Henares River course showing the location of samples. Black areas correspond to recent reservoirs. (D) Generalized geologic map of the study area showing the distribution of the main lithologic groups of bedrocks. 
sedimentary cover, up to $1600 \mathrm{~m}$ in thickness, which borders the southern edge of the Central System. The sedimentary sequence includes Permian, Triassic, Cretaceous and Paleogene strata. Permian strata include shale, sandstone and conglomerate, interbedded with andesitic and rhyolitic volcanics (the latter having plagioclase, amphibole, biotite and garnet phenocrysts). The Permian strata are overlain by Triassic Germanic facies (detrital Buntsandstein, dolomitic Muschelkalk and lutitic-evaporitic Keuper). The Cretaceous strata include sandstone, shallow marine limestone and dolostone, with gypsiferous strata at the top of the sequence. The Paleogene strata represent a continental synorogenic clastic wedge deposited following the Alpine build-up of the Central System and the Iberian Range, and during the initial configuration of the Tertiary Tajo Basin (Arribas and Arribas, 1991). The Paleogene strata include lacusrine carbonates overlain by clastic deposits (rudite and arenite) from prograding alluvial fans, representing the initial infilling of the Tajo Basin (Arribas et al., 1983).

The Iberian Range is a NW-SE trending Alpine mountain chain, about $400 \mathrm{~km}$ long, where Mesozoic sedimentary rocks unconformably overlie the preHercynian basement. The catchment area of the Henares River is located in the northwest sector of the Iberian Range, where the stratigraphic succession is mainly represented by Triassic (Germanic facies) and Jurassic shallow marine carbonates (micritic and dolomicritic microfacies). Cretaceous siliciclastic (quartzarenite and subarkose) and marine carbonate formations (dolostone and limestone) crop out along the southem border of the chain. The Triassic and Cretaceous deposits are similar to those of the Central System cover, although thicknesses are greater in the Iberian Range.

The Tajo Basin mainly contains continental Neogene clastics (fluvial fans) supplied from both the Central System and the Iberian Range, with compositions very similar to those of the Paleogene age. Evaporite and carbonate lacustrine (micritic microfacies) deposits, cap the Neogene sequence, and represent the distal facies of fans developed in an arid-subarid climate.

During Pliocene and Quaternary times the modern hydrography patterns were established. In addition, Pliocene erosion surfaces covered by clastic deposits occurred on several areas of the Central System (Portero et al., 1991; Tortosa et al., 1997).

\section{Climate and physiography of drainage basins}

The climate of the study area can be classified as continental Mediterranean. Mean annual precipitation varies from $750 \mathrm{~mm}$ in the mountainous area (northwestern sector) to less than $500 \mathrm{~mm}$ in the lowlands (southern sector). Mean annual temperature also differs from $7.5^{\text {to }} 12.5^{\circ} \mathrm{C}$, respectively (Table 1; I.G.N., 1991). As a function of these climatic parameters, weathering in the mountainous area may be considered as 'chemically moderate with frost action', whereas the lowlands are subjected to 'very slight weathering' (Wilson, 1969). Thin soil horizons are developed on metamorphic and sedimentary source rocks in the mountainous areas. Because of their steep slopes and torrential streams (mainly on the Central System), metamorphic and sedimentary rocks are removed by transport processes more rapidly than the rate at which weathering processes generate the deritus. Thus, erosion takes place under a weathering-limited denudation regime (Carson and Kirkby, 1972; Johnsson, 1993)

The total catchment area of the three main tributaries of the Henares River is $804.8 \mathrm{~km}^{2}$ (Table 1). Their drainage basins are contiguous, showing an elongated shape in a north-south trend. Maximum altitude is $2048 \mathrm{~m}$ (along Sorbe basin divide), and minimum altitude is about $740 \mathrm{~m}$ (to the confluences of the Sorbe River and Henares River). The proportions of the bedrock lithologies (gneiss, slateschist, limestone and sandstone) vary drastically from one basin to another (Tables 1 and 2). Sorbe, Bornova and Cañamares rivers have permanent flows, whereas their tributaries have mainly intermittent (seasonal) flows. The lower reaches of the three rivers flow across the Tertiary Plateau with a gentle topography. Unfortunately, there is not annual average data about the hydrological parameters controlling flow of the analyzed rivers. During the past two decades (19802000), dams have been built near the mouths of the all three drainage basins, controlling the flow along the lower racts. Details of the characteristics of the Sorbe, Bornova and Cañamares river basins are reported in Table 1. 

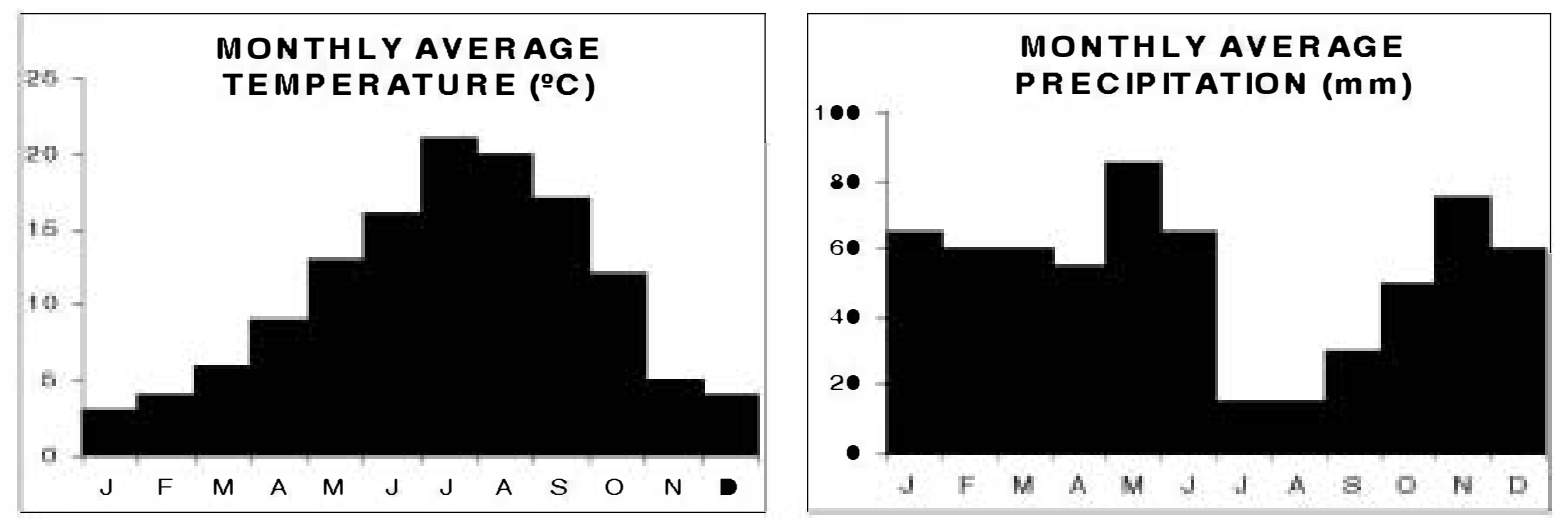

\begin{tabular}{|c|c|c|c|}
\hline & CAÑAMARES & BORNOVA & SORBE \\
\hline Catchment area $\left(\mathrm{km}^{2}\right)$ & 212.15 & 260.97 & 331.75 \\
\hline Max. Basin Length (m) & 38400 & 42800 & 50400 \\
\hline Min. altitude & 799 & 790 & 740 \\
\hline Mean slope (\%) & $9.47 \pm 9.03$ & $12.37 \pm 11.2$ & $15.09 \pm 13.5$ \\
\hline
\end{tabular}

\footnotetext{
${ }^{a}$ See also Table 2 for more details.
}

\section{Sampling and procedures}

As the main goal of this study is to observe the relationships between source area and the composition of sampled sediments, we have used a GIS (Ilwis v. 1.4) to quantify the most relevant source terrain characteristics. This characterization requires the combination of topography and geological parameters. Topographic information from topographic maps (scale: $1 / 100,000$ ) was used to produce a digital elevation model (DEM). This, in turn, was used to generate slope maps by filtering. Geological information concerning lithology of drainage basins was acquired from geologic maps (scale: 1/50,000 and
$1 / 200,000)$ and transformed by digitizing into raster map formats. This enabled the quantification of the area occupied by each lithologic group in the drainage basin, together with the median slope (Montesinos and Arribas, 1998). For each sand sample, the sub-basin is defined here as comprising portion of the drainage basin that is behind the sample site. Table 2 gives the surface area of each bedrock group for each sub-basin, together with the related slope.

We analyzed a total of 19 sand samples. Eight sand samples come from the Henares River main channel, collected from bars in the inner reaches of the channel or directly from active channel. Eleven stream sand 
Table 2

Occurrence of source lithology and mean slope of the analyzed sub-basins (Slp: mean slope)

\begin{tabular}{|c|c|c|c|c|c|c|c|c|c|c|c|c|c|c|c|c|c|c|c|c|c|c|c|c|c|c|c|c|c|c|}
\hline \multirow[t]{3}{*}{ Lithølogy } & \multicolumn{2}{|l|}{$\mathrm{C} 1$} & \multicolumn{2}{|l|}{$\mathrm{C} 2$} & \multicolumn{3}{|l|}{03} & \multicolumn{3}{|l|}{$\mathrm{C} 4$} & \multicolumn{2}{|l|}{ B 1} & \multicolumn{3}{|l|}{$\mathbf{2} 2$} & \multicolumn{3}{|l|}{$\mathbf{B} 3$} & \multicolumn{3}{|l|}{ B 4} & \multicolumn{3}{|l|}{$\mathrm{S} 1$} & \multicolumn{3}{|l|}{ s2 } & \multicolumn{3}{|l|}{ S3 } \\
\hline & \multirow{2}{*}{$\frac{\text { Area }}{\left(\mathrm{m}^{2}\right)(\%)}$} & \multirow{2}{*}{$\begin{array}{l}\text { Slp } \\
(\%)\end{array}$} & \multirow{2}{*}{$\frac{\text { Area }}{\left(\mathrm{m}^{2}\right)(\%)}$} & \multirow{2}{*}{$\begin{array}{l}\text { Slp } \\
(\%)\end{array}$} & \multicolumn{2}{|l|}{ Area } & \multirow{2}{*}{$\begin{array}{l}\text { Slp } \\
\%)\end{array}$} & \multicolumn{2}{|l|}{ Area } & \multirow{2}{*}{$\begin{array}{l}\text { Slp } \\
(\%)\end{array}$} & \multirow{2}{*}{$\frac{\text { Area }}{\left(\mathrm{km}^{2}\right)(\%)}$} & \multirow{2}{*}{$\begin{array}{l}\text { Slp } \\
(\%)\end{array}$} & \multirow{2}{*}{\multicolumn{2}{|c|}{$\frac{\text { Area }}{\left(\mathrm{m}^{2}\right)(\%)}$}} & \multirow{2}{*}{$\begin{array}{l}\text { Slp } \\
\%)\end{array}$} & \multirow{2}{*}{\multicolumn{2}{|c|}{$\frac{\text { Area }}{\left(\mathrm{km}^{2}\right)(\%)}$}} & \multirow{2}{*}{$\begin{array}{l}\text { Slp } \\
\%)\end{array}$} & \multirow{2}{*}{\multicolumn{2}{|c|}{$\frac{\text { Area }}{\left(\mathrm{km}^{2}\right)(\%)}$}} & \multirow{2}{*}{$\begin{array}{l}\text { Slp } \\
(\%)\end{array}$} & \multirow{2}{*}{\multicolumn{2}{|c|}{$\frac{\text { Area }}{\left(\mathrm{m}^{2}\right)(\%)}$}} & Slp & Area & & Slp & Area & & Slp \\
\hline & & & & & $\left(\mathrm{m}^{2}\right)$ & $\%)$ & & $\left(\mathbf{m}^{2}\right)$ & $(\%)$ & & & & & & & & & & & & & & & $\%)$ & $\left(\mathrm{m}^{2}\right)$ & $(\%)$ & $\%)$ & $\left(\mathrm{km}^{2}\right)$ & $(\%)$ & $(\%)$ \\
\hline $\begin{array}{l}\text { ANDESITES } \\
\text { Permian }\end{array}$ & $36 \quad 1.7$ & 84 & $36 \quad 18$ & 84 & 36 & 19 & 84 & 36 & 34 & 84 & -- & - & - & - & - & - & - & - & - & - & - & - & - & - & - & - & - & - & - & - \\
\hline $\begin{array}{l}\text { CLASTICS } \\
\text { (Congl., sst., mud) } \\
\text { Permian t• }\end{array}$ & 123.258 .1 & 73 & 1128559 & 74 & 99.6 & 52. & 7.1 & 62.8 & 602 & 58 & $100 \cdot 38.3$ & 79 & 23.6 & $24 \bullet$ & 9.2 & 767 & 32.3 & 84 & 868 & $35 \bullet$ & 83 & 87.3 & 26.3 & 83 & 33.5 & 13.2 & 65 & 7032 & 22.3 & 85 \\
\hline $\begin{array}{l}\text { CARBONATES } \\
\text { Jurassic \& Cretaceous }\end{array}$ & 19.19 & 12.8 & 19.195 & 12 & 19.1 & 101 & 12. & 12.5 & $12 \bullet$ & 13.9 & 16.162 & 15.7 & 12.2 & 12.4 & 16.5 & 16.1 & 68 & 15.7 & 16.1 & 65 & 15.7 & 39.2 & 11.8 & 91 & 31.3 & 12.4 & 83 & 39.21 & 12.5 & 9.2 \\
\hline $\begin{array}{l}\text { METAQUARTZITES } \\
\text { ondovician \& Silurian }\end{array}$ & 6330 & 13.6 & $63 \quad 31$ & 13.6 & 63 & 34 & 13.6 & 43 & 41 & 13.7 & 30.311 .6 & 20.6 & 15.3 & 15.5 & 206 & 30.3 & 12.8 & 20.6 & 30.3 & 12.2 & 20.6 & 32.9 & 9 & 23. & 303 & $12 \bullet$ & $23 \bullet$ & 32.91 & 10.5 & 23. \\
\hline SCHISTS Silurian & 10147 & 10.6 & 1015 & 10.6 & $1 \bullet 1$ & 53 & 10.6 & 101 & 97 & 106 & $-\quad-$ & - & - & - & - & - & - & - & - & - & - & - & - & - & - & - & - & - & - & - \\
\hline $\begin{array}{l}\text { GHEISS } \\
\text { Cambrian-ond ovician }\end{array}$ & 31.214 .7 & 11.2 & 31.215 .5 & 11.2 & 31.2 & 165 & 11.2 & 25 & 24 & 15.5 & 49.118 .8 & 11.2 & 78 & 79 & 13.2 & 49.1 & 20.7 & 11.2 & 49.1 & 19.8 & 11.2 & - & - & - & - & - & - & - & - & - \\
\hline SLATES & 18788 & 15.5 & 18793 & 15.5 & 187 & 9 & 15.5 & 85 & 22 & $15 \bullet$ & 65.425 & 15.5 & 39.6 & $4 \cdot 2$ & 160 & 65.4 & 27.5 & 15.5 & 65.4 & 26.4 & 15.5 & 1723 & 51.9 & 18.4 & 158 & 62.4 & 187 & 17235 & 548 & 184 \\
\hline
\end{tabular}


Table 3

Results of petrographic modal analysis

\begin{tabular}{|c|c|c|c|c|c|c|c|c|c|c|c|c|c|c|c|c|c|c|c|c|c|}
\hline & & & $\mathrm{C} 1$ & $\mathrm{C} 2$ & $\mathrm{C} 3$ & $\mathrm{C} 4$ & B1 & B2 & B3 & B4 & S1 & S2 & S3 & H1 & $\mathrm{H} 2$ & $\mathrm{H} 3$ & $\mathrm{H} 4$ & $\mathrm{H} 5$ & $\mathrm{H} 6$ & $\mathrm{H} 7$ & H8 \\
\hline \multirow[t]{33}{*}{ NCE } & \multirow{10}{*}{$\mathbf{e}$} & Quartz (single crystal) & 106 & 111 & 118 & 118 & 101 & 137 & 44 & 108 & 125 & 46 & 156 & 121 & 127 & 124 & 122 & 128 & 12 & 113 & 133 \\
\hline & & $\begin{array}{l}\text { P•lycrystalline quartz with } \\
\text { tect }\end{array}$ & 4 & 2 & 4 & 3 & 2 & 5 & $\bullet$ & 3 & 5 & 7 & , & 5 & 5 & 3 & 2 & $\bullet$ & 3 & 2 & 5 \\
\hline & & $\begin{array}{l}\text { Polycrystalline quartz without } \\
\text { tectonic fabric }\end{array}$ & $\bullet$ & 2 & - & 5 & $\bullet$ & - & $\bullet$ & $\bullet$ & - & - & - & 1 & 1 & - & 4 & 3 & 2 & 2 & $\bullet$ \\
\hline & & Quartz with evaperitic inclusions & 3 & 1 & 4 & - & - & - & - & $\bullet$ & - & - & $\bullet$ & 2 & - & - & 4 & 1 & 3 & - & 3 \\
\hline & & $\begin{array}{l}\text { Quartz in løw and medium } \\
\text { metamerphic grade rock fragm. }\end{array}$ & 38 & 48 & 53 & 24 & $5 \bullet$ & 37 & 19 & 56 & 26 & 2 & 33 & 36 & 46 & 58 & 57 & 61 & 46 & 58 & 48 \\
\hline & & Quartz in plut nic reck fragment & 2 & - & - & - & $\bullet$ & - & - & 1 & 1 & $\bullet$ & $\bullet$ & 1 & - & - & $\bullet$ & $\bullet$ & - & $\bullet$ & $\bullet$ \\
\hline & & Quartz in gneissic rock fragment & 15 & 16 & 16 & 1 & 3 & 6 & 1 & 9 & 2 & - & 8 & 4 & 5 & 4 & 10 & 10 & , & 6 & 5 \\
\hline & & Quartz in sandst & $\bullet$ & $\bullet$ & 2 & 2 & 1 & - & $\bullet$ & - & $\bullet$ & - & $\bullet$ & 1 & $\bullet$ & - & 6 & - & 4 & 6 & 12 \\
\hline & & $\begin{array}{l}\text { Quartz in } \\
\text { fragment }\end{array}$ & - & - & - & - & $\bullet$ & $\bullet$ & $\bullet$ & $\bullet$ & 1 & - & $\bullet$ & $\bullet$ & - & - & $\bullet$ & $\bullet$ & - & - & $\bullet$ \\
\hline & & Quartz with •vergrowth & $\bullet$ & $\bullet$ & $\bullet$ & - & $\bullet$ & - & $\bullet$ & $\bullet$ & $\bullet$ & $\bullet$ & $\bullet$ & $\bullet$ & $\bullet$ & $\bullet$ & $\bullet$ & - & $\bullet$ & 1 & 1 \\
\hline & \multirow[t]{5}{*}{$\mathrm{K}$} & $\mathrm{K}$-feldspar (single crystal) & 31 & 19 & 17 & 6 & 7 & 26 & 7 & 7 & 11 & 2 & 10 & 12 & 18 & 10 & 8 & 8 & 21 & 27 & 7 \\
\hline & & $\begin{array}{l}\mathrm{K} \text {-feldspar in low and medium } \\
\text { metamerphic rade reck fratm. }\end{array}$ & - & - & $\bullet$ & - & 4 & - & - & 2 & 1 & 1 & 1 & 2 & $\bullet$ & 2 & 1 & 1 & 1 & $\bullet$ & 2 \\
\hline & & $\begin{array}{l}\text { K-feldspar in plutenic reck } \\
\text { fragment }\end{array}$ & 2 & - & 1 & 1 & 3 & $\bullet$ & - & 1 & 1 & - & - & $\bullet$ & - & 2 & $\bullet$ & $\bullet$ & 1 & $\bullet$ & 1 \\
\hline & & $\begin{array}{l}\text { K-feldspar in gneissic reck } \\
\text { fragment }\end{array}$ & 4 & 4 & 2 & - & $\bullet$ & $\bullet$ & $\bullet$ & $\bullet$ & $\bullet$ & $\bullet$ & $\bullet$ & 2 & 1 & $\bullet$ & $\bullet$ & 3 & - & $\bullet$ & $\bullet$ \\
\hline & & $\mathrm{K}$-feldspar in sandstone & - & - & $\bullet$ & - & - & $\bullet$ & $\bullet$ & 1 & - & - & - & $\bullet$ & $\bullet$ & $\bullet$ & $\bullet$ & $\bullet$ & - & $\bullet$ & 1 \\
\hline & \multirow[t]{5}{*}{$\mathbf{P}$} & Plagieclase (single crystal) & 19 & 13 & 17 & $4 \bullet$ & 24 & 14 & 7 & 25 & 4 & 1 & 2 & 6 & 10 & 16 & 15 & 22 & 15 & 18 & , \\
\hline & & $\begin{array}{l}\text { Plagioclase in low and medium } \\
\text { metamorphic grade rock fratm }\end{array}$ & 5 & 5 & 3 & 2 & 5 & 1 & 3 & 7 & 1 & 1 & 2 & 5 & 13 & 5 & 3 & 6 & 6 & 3 & 1 \\
\hline & & $\begin{array}{l}\text { Plagioclase in plutenic rock } \\
\text { fragment }\end{array}$ & 4 & - & - & - & - & $\bullet$ & - & 2 & - & $\bullet$ & $\bullet$ & 3 & 1 & 1 & 2 & $\bullet$ & 3 & 1 & $\bullet$ \\
\hline & & $\begin{array}{l}\text { Plagiøclase in gneissic reck } \\
\text { fragment }\end{array}$ & 6 & 6 & 2 & 1 & 4 & 8 & $\bullet$ & 1 & - & - & $\bullet$ & 1 & 1 & 1 & 2 & 1 & - & $\bullet$ & $\bullet$ \\
\hline & & Plagioclase in sandstene & - & $\bullet$ & $\bullet$ & 2 & $\bullet$ & - & - & - & - & - & - & $\bullet$ & - & - & $\bullet$ & $\bullet$ & - & $\bullet$ & 2 \\
\hline & \multirow[t]{4}{*}{ M } & Biotite (single crystal) & 2 & - & 1 & 5 & $\bullet$ & 2 & 1 & 1 & - & $\bullet$ & - & 2 & - & 2 & $\bullet$ & 1 & - & - & - \\
\hline & & Muscovite (single crystal) & $\bullet$ & $\bullet$ & 2 & - & 1 & 1 & - & 1 & $\bullet$ & $\bullet$ & $\bullet$ & 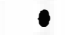 & $\bullet$ & 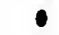 & 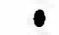 & 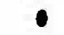 & $\bullet$ & $\bullet$ & - \\
\hline & & Chlorite (single crystal) & 2 & - & - & - & - & 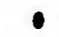 & - & 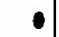 & $\bullet$ & - & $\bullet$ & - & $\bullet$ & 1 & $\bullet$ & - & 1 & 2 & - \\
\hline & & $\begin{array}{l}\text { Mica in lew and medium } \\
\text { metamorphic grade rock fragm. }\end{array}$ & 1 & 3 & $\bullet$ & 4 & 2 & 1 & 1 & $\bullet$ & 1 & - & $\bullet$ & 1 & $\bullet$ & 2 & 1 & 1 & 2 & - & 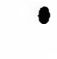 \\
\hline & \multirow[t]{8}{*}{$\mathrm{L}$} & Schist & 16 & 3 & 13 & 4 & 4 & 5 & 4 & 7 & 5 & 19 & 6 & 6 & $\bullet$ & 5 & 4 & 8 & 4 & 5 & 2 \\
\hline & & Phyllite & 15 & 13 & 12 & 44 & 28 & 38 & 11 & 25 & 79 & 188 & 47 & 31 & 26 & 34 & 2 & 23 & 18 & 13 & 2 \\
\hline & & Siltstone with carbnate cement & $\bullet$ & 1 & $\bullet$ & - & 1 & 2 & $\bullet$ & 2 & $\bullet$ & $\bullet$ & $\bullet$ & $\bullet$ & 3 & 1 & $\bullet$ & $\bullet$ & $\bullet$ & 1 & 3 \\
\hline & & $\begin{array}{l}\text { Siltst ne withøut carbønate } \\
\text { cement }\end{array}$ & 1 & - & 1 & 4 & 3 & - & - & 1 & 6 & 2 & $\bullet$ & 5 & 2 & 5 & 5 & $\bullet$ & 1 & - & 2 \\
\hline & & Shale & 1 & - & 3 & 6 & 1 & - & - & 1 & 2 & 2 & $\bullet$ & 2 & - & - & $\bullet$ & $\bullet$ & - & 1 & - \\
\hline & & Metavelcanic & 3 & 4 & - & 4 & - & - & - & - & - & - & - & - & - & 1 & 3 & - & 1 & - & - \\
\hline & & Velcanic with micr lithic texture & - & - & - & 1 & $\bullet$ & - & - & $\bullet$ & - & $\bullet$ & - & $\bullet$ & $\bullet$ & $\bullet$ & - & 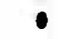 & - & 1 & $\bullet$ \\
\hline & & $\begin{array}{l}\text { Volcanic with felsitic granular } \\
\text { texture }\end{array}$ & - & - & - & 2 & - & - & - & $\bullet$ & $\bullet$ & $\bullet$ & - & 2 & - & - & $\bullet$ & $\bullet$ & 1 & 1 & $\bullet$ \\
\hline & at & Heavy minerals (unspecified) & 1 & 8 & 3 & 1 & 36 & 3 & - & 2 & 11 & , & 18 & $\bullet$ & 3 & 5 & 7 & 4 & 4 & - & 8 \\
\hline
\end{tabular}


Table 3 (continued)

\begin{tabular}{|c|c|c|c|c|c|c|c|c|c|c|c|c|c|c|c|c|c|c|c|c|c|c|c|}
\hline & & & $\mathrm{C} 1$ & $\mathrm{C} 2$ & $\mathrm{C} 3$ & $\mathrm{C} 4$ & B 1 & $\mathbf{B} 2$ & B3 & B4 & S1 & S2 & S3 & $\mathrm{H} 1$ & $\mathrm{H} 2$ & H3 & $\mathrm{H} 4$ & $\mathrm{H} 5$ & H6 & $\mathrm{H} 7$ & H8 & & \\
\hline \multirow[t]{13}{*}{$\mathrm{CE}$} & \multirow{13}{*}{ CAL } & Micritic limestene & - & 3 & $\bullet$ & $\bullet$ & $\bullet$ & - & 24 & 1 & 3 & $\bullet$ & 2 & 7 & 7 & $\bullet$ & 5 & 1 & 3 & 10 & 19 & & \\
\hline & & Wackest $\bullet$ e + Biømicrite & 1 & 1 & 1 & - & - & 3 & $\bullet$ & - & - & $\bullet$ & 2 & $\bullet$ & - & - & 0 & - & $\bullet$ & $\bullet$ & 1 & & \\
\hline & & Grainstone & 2 & - & 1 & - & - & 2 & - & - & - & $\bullet$ & 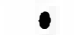 & $\bullet$ & 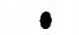 & - & 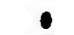 & $\bullet$ & $\bullet$ & $\bullet$ & 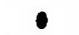 & & \\
\hline & & Sparitic limestene & 2 & 3 & 2 & 2 & 4 & 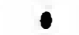 & 112 & 7 & 2 & $\bullet$ & - & 5 & 3 & - & 2 & 3 & 2 & 2 & 1 & & \\
\hline & & Microsparitic limestone & 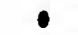 & $\bullet$ & - & 2 & $\bullet$ & 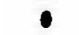 & 16 & 1 & 1 & $\bullet$ & - & $\bullet$ & 1 & $\bullet$ & 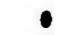 & $\bullet$ & 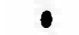 & 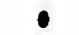 & 3 & & \\
\hline & & Calcite (single crystal) & $\bullet$ & $\bullet$ & 2 & 4 & 4 & 2 & 19 & 2 & 2 & $\bullet$ & 3 & 2 & 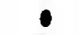 & $\bullet$ & 1 & $\bullet$ & 4 & 2 & 2 & & \\
\hline & & Fossil & $\bullet$ & 3 & $\bullet$ & 5 & 2 & 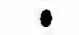 & $\bullet$ & 1 & $\bullet$ & $\bullet$ & - & 2 & 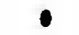 & $\bullet$ & 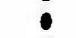 & 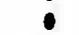 & - & 3 & - & & \\
\hline & & Silty-arenitic limestene & 1 & 3 & 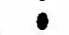 & 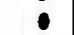 & 1 & 3 & 2 & 3 & 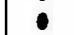 & - & 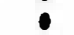 & 5 & 4 & 2 & 2 & 1 & 2 & 8 & 2 & & \\
\hline & & Dolomicrite & 4 & 10 & 6 & 4 & 3 & 2 & 4 & , & 2 & - & - & 2 & 7 & 4 & 1 & 1 & 5 & 3 & 6 & & \\
\hline & & Dolesparite & 8 & 4 & 8 & 1 & 1 & 2 & 10 & 7 & $\bullet$ & - & - & 11 & 7 & 6 & 3 & 7 & 6 & 3 & 11 & & \\
\hline & & Dolømite (single crystal) & - & 2 & 1 & 1 & 4 & - & 10 & $\bullet$ & 1 & $\bullet$ & 1 & 2 & 1 & $\bullet$ & 4 & 1 & 3 & 3 & 4 & & \\
\hline & & Silty-arenitic dolestone & 1 & 3 & - & 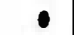 & $\bullet$ & 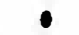 & 2 & 3 & 1 & $\bullet$ & $\bullet$ & 5 & 3 & 3 & 2 & $\bullet$ & 6 & 2 & 1 & & \\
\hline & & Dedolømite & - & 5 & 3 & - & - & $\bullet$ & 3 & 2 & 1 & - & - & 7 & 3 & 3 & 2 & 3 & 3 & 3 & 3 & & \\
\hline $\mathrm{Cl}$ & & Biøclast & $\bullet$ & - & $\bullet$ & $\bullet$ & $\bullet$ & $\bullet$ & $\bullet$ & $\bullet$ & $\bullet$ & $\bullet$ & $\bullet$ & $\bullet$ & - & - & 1 & $\bullet$ & $\bullet$ & $\bullet$ & $\bullet$ & & \\
\hline \multirow[t]{16}{*}{$\mathrm{NCl}$} & & Fe-xid concretion & $\bullet$ & 4 & 2 & - & 1 & $\bullet$ & $\bullet$ & 1 & 3 & 2 & 2 & 1 & $\bullet$ & $\bullet$ & 1 & 2 & $\bullet$ & $\bullet$ & $\bullet$ & \multirow{2}{*}{\multicolumn{2}{|c|}{ Henares }} \\
\hline & & Alterite & $\bullet$ & $\bullet$ & $\bullet$ & 1 & $\bullet$ & $\bullet$ & $\bullet$ & - & 2 & $\bullet$ & - & $\bullet$ & $\bullet$ & - & 0 & $\bullet$ & - & - & - & & \\
\hline & & Total & 300 & 300 & 300 & 300 & 300 & 300 & 300 & 300 & 300 & 300 & 300 & 300 & 300 & 300 & 300 & 300 & 300 & 300 & 300 & $\mathrm{X}$ & sd \\
\hline & & $\mathrm{m}$ & 55.8 & 61.8 & 66.1 & $5 \bullet .2$ & 59.6 & 61.4 & 21.5 & $5 \quad 59.0$ & 54.8 & 22.8 & 30.4 & 55.7 & 759.9 & 64.1 & 68.6 & $6 \quad 68.5$ & $5 \quad 62.1$ & 61.7 & 69.2 & 63.7 & \pm 4.6 \\
\hline & & $\mathrm{F}$ & 24.1 & 16.5 & 14.4 & 18.0 & 18.1 & 16.7 & 5.7 & 15.6 & 6.4 & $\begin{array}{l}2.7 \\
+\quad 1.7\end{array}$ & 5.4 & 10.5 & $5 \quad 14.8$ & 12.8 & 10.7 & 714.0 & 16. & 16.4 & 7.9 & 12.9 & \pm 2.9 \\
\hline & & $\mathrm{Lt}$ & 20.1 & 21.8 & 19.5 & 31.8 & 22.3 & 21.8 & 72.8 & 25.4 & 38.9 & 75.4 & 24.3 & 33.8 & 25.3 & 23.1 & 20.7 & $7 \quad 17.5$ & $5 \quad 21.8$ & 21.8 & 22.9 & 23.4 & \pm 4.6 \\
\hline & & $\mathrm{Lm}$ & 56.4 & 27.6 & 47.2 & 57.1 & 57.1 & 72.9 & 6.9 & 44.4 & 80.0 & 98.1 & 89.8 & 39.4 & $4 \quad 37.7$ & 60.9 & 44.4 & $4 \quad 64.6$ & $6 \quad 37.3$ & 29.5 & 6.5 & $4 \bullet .0$ & \pm 18.1 \\
\hline & & $\mathrm{Lv}$ & 5.5 & 6.9 & 0. & 8.3 & $\bullet .0$ & 0 & 0.0 & $\quad 0.0$ & 0.0 & 0 & 0.0 & 2.1 & 10 & 1.6 & 5.6 & 60 & $\quad 3.4$ & $\quad 3.3$ & 0 & 2. & \pm 2.1 \\
\hline & & Ls & 38.2 & 65.5 & 52.8 & 34.5 & 42.9 & 27.1 & 93.1 & 55.6 & 20.0 & 1.9 & 10.2 & 58.5 & 62.3 & 37.5 & 50.0 & 35.4 & $4 \quad 59.3$ & 67.2 & 93.5 & 58.0 & \pm 18.3 \\
\hline & & $\mathrm{Lm}$ & 59.6 & 29.6 & 47.2 & 62.3 & 57.1 & 72.9 & 6.9 & 44.4 & 80.0 & 98.1 & 89.8 & 40.2 & $2 \quad 37.7$ & 61.9 & 47.1 & 164.6 & 638.6 & 30.5 & 6.5 & 40.9 & \pm 18.3 \\
\hline & & Lss & 3.8 & 1.9 & 7.5 & 13. & 8.9 & $\quad 3.4$ & 0.0 & 5.6 & 7.6 & 1.9 & 0.0 & 7.6 & $6 \quad 7.2$ & $\quad 9.5$ & 9.8 & 80 & $\quad 1.8$ & 3.4 & 8.1 & 5.9 & \pm 3.7 \\
\hline & & Lsc & 36.5 & 68.5 & 45.3 & 24.7 & 33.9 & 23.7 & 93.1 & 50.0 & 12.4 & 10.0 & 10.2 & 52.2 & 255.1 & 28.6 & 43.1 & 135.4 & 459.6 & 66.1 & 85.5 & 53.2 & \pm 18. \\
\hline & & Lss & 9.5 & 2.6 & 14.3 & 34.5 & 20.8 & 12.5 & ८. & 10.0 & 38.1 & 100.0 & e.e & 12.7 & 11.6 & 25. & 18.5 & e.e & 2.9 & 4.9 & 8.6 & 10.5 & \pm 8.3 \\
\hline & & Lsd & 61.9 & 63.2 & 64.3 & 20.7 & 33.3 & 25. & 14.4 & 452.5 & 23.8 & 0 & 16.7 & 49.1 & 148.8 & 66.7 & 44.4 & 470.6 & $\begin{array}{ll}6 & 65.7\end{array}$ & 34.1 & 43.1 & 52.8 & \pm 13.3 \\
\hline & & Ls1 & 28.6 & 34.2 & 21.4 & 44.8 & 45.8 & 62.5 & 85.6 & 37.5 & 38.1 & $\bullet$ & 83.3 & 38.2 & 239.5 & 8.3 & 37.0 & - 29.4 & $4 \quad 31.4$ & $61 . \bullet$ & 48.3 & 36.6 & \pm 15.3 \\
\hline & & $P / F$ & 0.48 & $\bullet .51$ & $\bullet .52$ & 0.87 & 0.7 & 0.47 & $\bullet .59$ & 0.76 & 0.28 & $\bullet .4 \bullet$ & $\bullet .27$ & 0.48 & 0.57 & $\bullet .62$ & .71 & 0.71 & $\bullet .51$ & $\bullet .45$ & e.52 & $\bullet .6$ & \pm \\
\hline
\end{tabular}



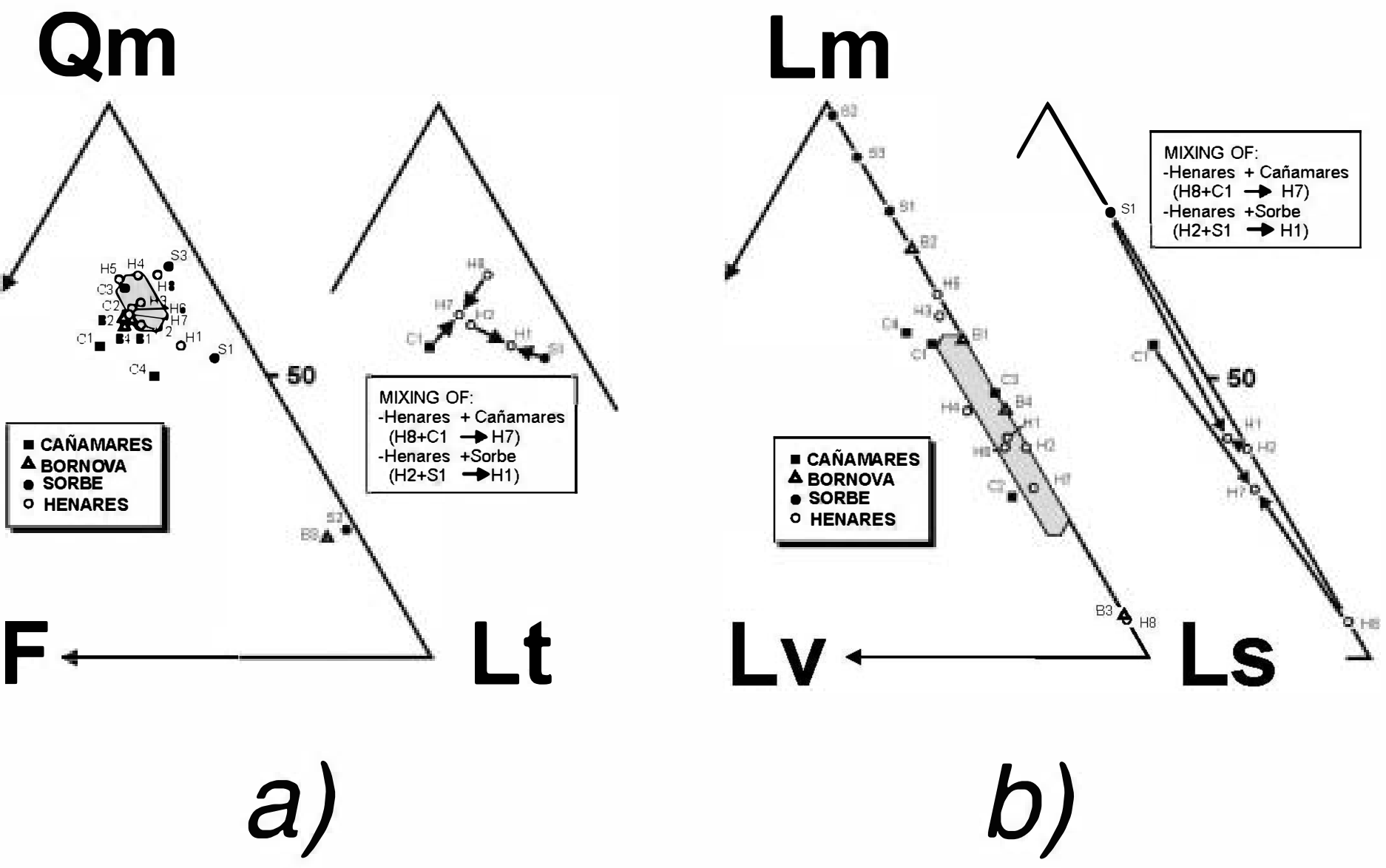

Fig. 2. (a) QmFLt ternary diagram show ing sand composition of samples from the Cañamares, Bornova, Sorbe and Henares rivers. The shaded field corresponds to the Henares variation field constructed using the standard deviation of Henares samples. Additional diagram emphasizes the mix ing process between the Henares and Cañamares sands, and between the Henares and Sorbe sands. (b) LmLvLsternary diagram show ing sand composition of samples from the Cañamares, Bornova, Sorbe and Henares rivers. The shaded field corresponds to the Henares variation field constructed using the standard deviation of Henares samples. Additional diagram emphasizes the mix ing process between the Henares and Cañamares sands, and between the Henares and Sorbe sands. 


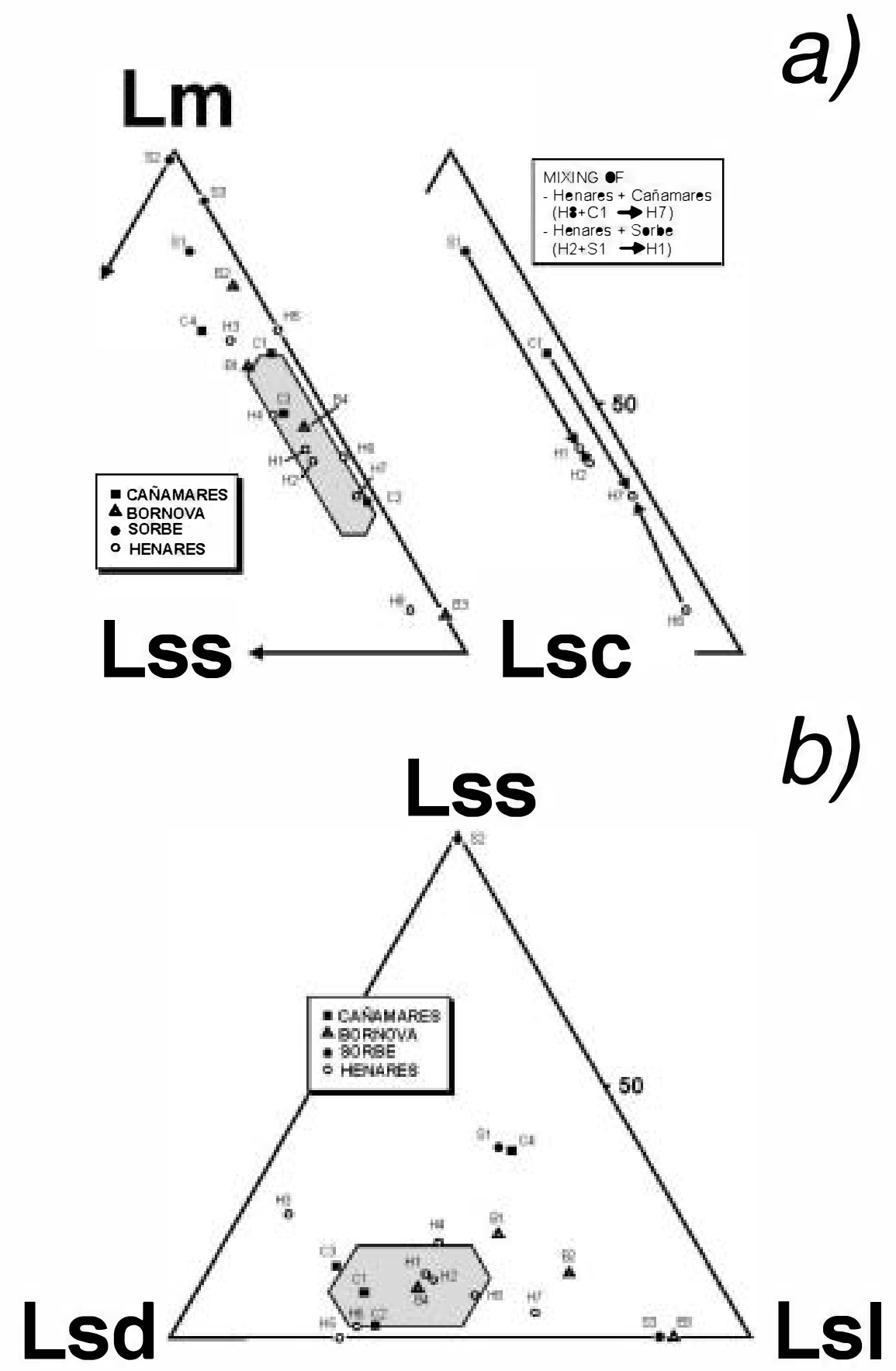

Fig. 3. (a) LmLssLsc temary diagram showing sand composition of samples from the Cañamares, Bornova, Sorbe and Henares rivers. The shaded field corresponds to the Henares variation field constructed using the standard deviation of Henares samples. Additional diagram emphasizes the mixing process between the Henares and Cañamares sands, and between the Henares and Sorbe sands. (b) LssLsdLsl ternary diagram showing sand composition of samples from the Cañamares, Bornova, Sorbe and Henares rivers. The shaded field corresponds to the Henares variation field constructed using the standard deviation of Henares samples. 
samples come from the three main tributaries of the Henares River, the Bornova River (four samples), the Cañamares River (four samples), and the Sorbe River (three samples). Following Ingersoll et al. (1993), sampling of sands of the Henares River and its tributaries, may be considered as second- and firstorder, respectively.

All sand samples were washed using $\mathrm{H}_{2} \mathbf{O}_{2}$ to remove clays and organic matter, air-dried and sieved (using 1-phi intervals). The $\mathbf{0 . 2 5}-\mathbf{0 . 5 0} \mathrm{mm}$ size fraction was used to prepare thin sections. Each thin section was etched and stained using HF and sodium cobaltinitrite for feldspars, and alizarine and potassium Fe-cyanide for carbonate grains. Three hundred points were counted on each thin section using the Gazzi-Dickinson method (Ingersoll et al., 1984; Zuffa, 1985). Point-count raw results and recalculated parameters are shown in Table 3 and plotted in Figs. 2 and 3 .

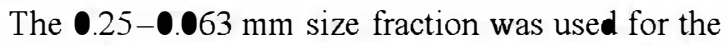
heavy mineral separation by gravity settling in bromoform (density $2.89 \mathrm{~g} / \mathrm{cm}^{3}$ at $2 \mathbf{0}^{\circ} \mathrm{C}$ ). Magnetite was separated from this size fraction magnetically using a bar magnet; while the remaining mineral groups were concentrated by multiple passes of the fraction through a Frantz Isodynamic magnetic separator to concentrate the other five fractions $(0.2 ; 0.5 ; 1.0 ; 1.5$ and $>1.5 \mathrm{~A}$ ), and to facilitate the mineral identification (Parfenoff et al., 1970). Heavy mineral grains of nine selected samples (one for Cañamares, two for Bornova, two for Sorbe, and four for Henares rivers) were analyzed microscopically. Five-hundred grains were determined (100 grains per each magnetic fraction) by the strip counting procedure. Finally, grain percentages for each sample, averaged from the fraction weights, were computed. Identified species and percentages of heavy minerals are summarized in Table 4.

\section{Grain types}

The major components of the Henares River sands, and of its tributaries, are: monomineralic quartz and feldspar grains, metamorphic rock fragments and sedimentary rock fragments (Table 3 ). Using the Gazzi-Dickinson method, these grains types are tabulated using the following main categories.

\subsection{Quartz}

This component occurs as single monocrystalline grains, fine-grained polycrystalline grains, and as coarse-grained (phaneritic) rock fragments (sedimentary, metamorphic and rare plutonic rock fragments). Samples include also quartz with evaporite inclusions, recycled from the Triassic (lutitic-evaporitic Keuper) sequences of the Iberian Range; and well-rounded monocrystalline quartz grains with abraded quartzcement overgrowth.

\subsection{Feldspars}

$\mathrm{K}$-feldspar is subordinate in all samples, and generally does not exceed $8 \%$. Plagioclase is generally more abundant than $\mathrm{K}$-feldspar, but does not exceed $11 \%$ (Table 3). Both plagioclase and K-feldspar occur in coarse-grained (phaneritic) sedimentary, metamorphic and rare plutonic rock fragments.

\subsection{Lithic fragments}

This category includes only fine-grained (aphanitic) fragments (Table 3), because the coarse-grained (phaneritic) rock fragments were not counted as rock fragments, but assigned to their respective monomineralic categories (i.e. quartz, feldspars, micas) depending on which crystal was encountered at the cross-hair (see Table 3). However, the abundance of phaneritic rock fragments can be recalculated from the Gazzi-Dickinson database (e.g. Critelli and Le Pera, 1994).

\subsubsection{Metamorphic Lithic Fragments (Lm)}

Fine-grained metamorphic fragments include slate/ phyllite and fine-grained schist. These grains range from 98.1 to $6.5 \%$ of lithic grains ( $\mathrm{LmLvLs} \% \mathrm{Lm}$ ). Gneissic, amphibolitic and schistose fragments are present, but are not counted as part of the metamorphic lithic fragment categories.

\subsubsection{Sedimentary Lithic Fragments (Ls)}

These grains range from 93.5 to $1.9 \%$ of lithic grains (LmLvLs\%Ls) and include a great variety of types, including both siliciclastic and carbonate lithic fragments. The siliciclastic lithics (Lss) consist of shale fragments, carbonate-cemented siltstones and siltstones with siliciclastic or ferruginous matrix. 
Table 4

Heavy mineral distributions (frequency percent) from Cañamares (C). Bornova (B), Sorbe (S) and Henares $(\mathrm{H})$ rivers

\begin{tabular}{|c|c|c|c|c|c|c|c|c|c|}
\hline Minerals & $\mathrm{C} 2$ & $\mathrm{~B} 1$ & $\mathrm{~B} 2$ & S1 & $\mathrm{S} 2$ & $\mathrm{H} 1$ & H5 & $\mathrm{H} 7$ & H8 \\
\hline Zircon & 0.0 & 0.9 & 11.3 & 0.7 & 1.2 & 9.0 & 1.1 & 1.0 & 0.5 \\
\hline Tourmaline & 6.7 & 2.3 & 5.0 & 2.0 & $\bullet .6$ & 2.9 & 4.1 & 4.5 & 8.2 \\
\hline Apatite & 2.6 & 5.5 & 0.0 & 3.2 & 0.9 & 8.9 & 3.2 & 3.5 & 1.7 \\
\hline $\mathrm{Ca}-\mathrm{Mg}$ carbonate & 22.6 & 1.3 & 0.3 & 5.6 & 0.2 & 5.8 & 0.7 & 1.8 & 17.3 \\
\hline Garnet & 14.2 & 34.8 & 0.2 & $\bullet .8$ & 0.0 & 4.3 & 23.6 & 10.5 & 14.4 \\
\hline Staurolite & 8.6 & 3.5 & 1.0 & 14.9 & 1.2 & 11.6 & 3.7 & 11.5 & 12.9 \\
\hline Kyanite & 0.5 & 3.9 & 9.9 & 0.0 & 0.0 & 5.3 & 3.4 & 2.5 & 2.3 \\
\hline Sphene & 3.6 & $\bullet .2$ & $\bullet .8$ & $\bullet .8$ & 0.0 & 1.1 & 0.3 & 0.0 & 0.0 \\
\hline Epidote group & 0.0 & 0.9 & 0.6 & 0.0 & 0.0 & $\bullet .6$ & 0.9 & 3.2 & 2.8 \\
\hline Ca-amphibole & 0.0 & 0.0 & 0.0 & $\bullet .0$ & 0.0 & 0.4 & 1.3 & 0.5 & 0. \\
\hline Transparent micaceous & 0.7 & $\bullet .0$ & 1.2 & $\bullet .0$ & 26.1 & 0.0 & 1.5 & 0.8 & - .4 \\
\hline Altered & 1.8 & 2.0 & 7.5 & 6.5 & 4.9 & 4.3 & 2.6 & 4.2 & 6. \\
\hline Polymineral aggregates & 3.3 & 1.9 & 5.0 & 1.3 & 2.2 & 3.6 & 5.3 & 6.0 & 1.7 \\
\hline Opaques & 30.5 & 37.9 & 24.3 & 62.7 & 61.7 & 30.9 & 36.7 & 45.5 & 27.5 \\
\hline Total & 100.0 & 100.0 & 100.0 & 100.0 & 100.0 & 100.0 & 100.0 & 100.0 & 100.0 \\
\hline
\end{tabular}

The total carbonate lithic fragments (Lsc) comprise diverse proportions of carbonate extrabasinal grains (CE; Zuffa 1985). The Lsc category is common in all the samples taken from the ributaries (except for upstream Sorbe sand, S2), and in the Henares sand. The Lsc category has been differentiated into two subgroups, namely Lsd (dolomicrite, dolosparite, single crystal of dolomite, silty-arenitic dolostones and dedolomite grains) and Lsl (diverse limestone types, single crystals of calcite, fossils, silty-arenitic limestones).

\subsubsection{Volcanic lithic fragments (Lv)}

A few grains of highly altered volcanic lithic fragments are present in some samples of the Cañamares and Henares River sands. Unaltered volcanic grains were subdivided using their textural attributes (e.g. Critelli and Ingersoll, 1995) into microlitic-texture and felsitic granular-texture fragments.

\subsection{Other counted grains}

These include micas and chlorite (single or polycrystalline), heavy minerals and penecontempor- aneous in rabasinal grains. The latter are caliche and ferricrete fragments, both of pedogenetic origin. We consider as "alterite" all grains lacking recognizable diagnostic composition and texture because of alteration.

\subsubsection{Heavy minerals}

In addition to gross composition of sand, a specific point-count analysis of the heavy mineral assemblages was carried out. Point-count results are tabulated in Table 4. All samples have abundant opaque minerals. Transparent non-micaceous species are: andalusite, apatite, baryte, $\mathrm{Ca}-\mathrm{Mg}$ carbonate (mainly dolomite), calcic amphibole (green homblende), epidote (including zoisite), Fe-carbonate (siderite), garnet (dominantly colourless/pink; yellow/brown grains found occasionally), kyanite, rutile (including anatase and brookite), sillimanite, staurolite, sphene, tourmaline (brown and blue varieties) and zircon Many of these species are present in only trace amounts: major minerals are andalusite, apatite, $\mathrm{Ca}-\mathrm{Mg}$ carbonate, garnet, kyanite, tourmaline and zircon 


\section{Modal sand composition of the tributaries}

\subsection{Cañamares River}

Sands from the Cañamares River drainage basin make a cluster on a QmFLt diagram, with monocrystalline quartz contents ranging from $5 \mathbf{0 . 2}$ to $66.1 \%$. Evolution of sand composition through the river course (from $\mathrm{C} 4$ to $\mathrm{Cl}$; Fig. 1C) is mainly related to the change in the relative proportion of bedrock lithologies in the corresponding sub-basins. Thus, the greatest content of lithic components appears upstream (C4), where sedimentary (carbonate) sources dominate over metamorphic (schist and slate) and volcanic sources (Table 2). However, the relative proportion of these source rocks does not correspond to the lithic grain proportions in the sand, which contains more metamorphic lithics than sedimentary lithics (Figs $2 \mathrm{~b}$ and $3 \mathrm{a})$. An important increase in quartz content is observed in sample C3, probably due to the increase of the gneissic outcrop area $(16.5 \%)$. This gneiss outcrop generates greater quantities of quartzose sediment, thus diluting lithic contributions and masking any race of volcanic lithics from Permian andesite outcrops. The progressive loss of monocrystalline quartz in the downstream zone (samples $\mathrm{C} 2-\mathrm{C} 1$ ), although nearly insignificant, may be related to recycling, either of Mesozoic sedimentary rocks cropping out in the contact with the Tajo Basin, or of Tertiary and Quaternary clastic deposits (lithic arkoses and subarkoses) from the above-mentioned basin. The erosion of Mesozoic carbonates, close to the Tajo Basin (samples C3, C2 and Cl in Fig. 3a and b), is also marke by local increases in dolomitic grain content. The presence of volcanic lithic fragments in these samples is associated with Permian volcaniclastic deposits outcropping in this area, included in the 'clastic' group shown in Fig. 1D and Table 2. Near to the confluence of the Cañamares River (sample Cl) and the Henares River, sands contain increased amounts of metamorphic lithic fragments. This increase could be related to homogenization processes affecting sand composition during the last tract of the river course (Ingersoll et al., 1993). Sedimentary source rocks in the upper reaches seem to be relatively unproductive in lithics; although the sedimentary outcrop area in the C4 sub-basin comprises $72 \%$, sedimentary lithics only form $34.5 \%$ of total lithics.
However, sedimentary sources located downstream produce greater amounts of lithics $(65.5 \%$ with respect to the total lithic fragments), with a total outcrop area in the sub-basin of $65.4 \%$ (sample C2; Table 2). This is probably related to the low relief of the upstream areas to the north, associated with sedimentary outcrops with near-horizontal strata (compare Fig. $1 \mathrm{~B}$ and D). P/F values are constant through the middle and downstream river courses with ratios near 0.5 (Table 2). This ratio value is comparable with that of gneissic bedrock, which ranges between 0.4 and 0.55 (Navidad, 1978). However, the $P / F$ ratio upstream, prior to the erosion of the gneissic area (sample C4), is $\mathbf{0 . 8 7}$, reflecting input of plagioclase from Permian andesites.

The heavy mineral suite from the Cañamares River (sample C2) reflects the contribution of sedimentary, metasedimentary and gneissic source rocks. Characteristic sedimentary minerals include $\mathrm{Ca}-\mathrm{Mg}$ carbonate $(22.6 \%)$ and baryte $(2.2 \%)$ while garnet $(14.2 \%)$ and staurolite $(8.6 \%)$ represent the metamorphic provenance (Table 4).

\subsection{Bomova River}

Sands from this drainage basin plot in a very tight cluster on the QmFLt diagram, with high em content (near 60\%) and a slight dominance of lithic fragments over feldspar content (Fig. 2a). However, sample B3 plots away from the cluster, due to high amounts of lithic fragments (72.8\%). This sample was collected near the entrance of the Bornova River into the Tajo Basin, where a narrow pass through Mesozoic carbonate rocks leads to erosion and supply of carbonate components (Figs. 2b, 3a and b). The two dominant sources in the drainage basin are sedimentary and metamorphic, reflected by the plot of all samples along the LmLs line (Fig. 2b). Sedimentary lithic grains are mainly carbonate (limestone and dolostone fragments, Fig. 3b). Phyllite grains are the most abundant metamorphic lithics $(\mathrm{Lm})$. The greatest content of phyllite fragments occurs upstream $(\mathbf{7 2 . 9 \%}$ of total lithics in sample B2), where the corresponding subbasin displays an outcrop area of this lithology of about $40 \%$ (slates in Table 2). The great peak in the content of carbonate lithics (sample B3), is progressively diluted in the distal stream (samples B4 and B 1) as a result of homogenization processes, similar to 
that described for the Cañamares River. In the Bornova drainage basin, upstream sedimentary sources generate low quantities of lithics compared with downstream sands, as in the Cañamares drainage basin. $P / F$ values are near 0.5 in upstream sand (samples $\mathbf{B} 2$ and B3). These values are low compared with those from gneissic rocks in the source area (0.55-0.73; Navidad, 1978). The relative increase of $\mathrm{K}$-feldspar in the sands could be related to supply from Triassic sandstones cropping out in the north of the drainage basin.

The most upstream Bornova sand sample (B2) contains an andalusite (18\%)-sillimanite (14.2\%)zircon $(11.3 \%)$ suite. Kyanite $(9.9 \%)$ and tourmaline $(5 \%)$ are other significant species, with $\mathrm{Ca}-\mathrm{Mg}$ carbonate, epidote, $\mathrm{Fe}$-carbonate, garnet, sphene and staurolite present in trace amounts (Table 4). A change in the heavy mineral association takes place a few kilometers downstream (sample B1) where garnet (both colorless and pink types, $34.8 \%)$, apatite $(5.5 \%)$, kyanite $(3.9 \%)$ and staurolite $(3.5 \%)$ are the main constituents of the transparent non-micaceous population. The sharp contrast is related to an increase in the outcrop of gneissic rocks in the lower reaches of the river (Fig. 1).

\subsection{Sorbe River}

Samples from this drainage basin show feldspar contents lower than 6.4\% (QmFLt diagram), differing from Cañamares and Bornova river samples. Coarsegrained metamorphic rocks (schist, gneiss) are not present in the hinterland, and thus little quartzo-feldspathic supply can be expected. In the upstream sand (sample S2) this effect is extreme, as shown by the total lithics of $75.4 \%$ (Lt in Fig. 2a). The lithic fragments are metamorphic, contributed by a slate outcrop area of $62.4 \%$. In this portion of the river course, sedimentary lithic fragments are scarce, and volcanic lithics are absent. As described for the Cañamares and Bornova drainage basins, sedimentary sources located in the north of the basin produce little sand-sized deritus, even though they have a considerable outcrop area (25.6\% in S2 sub-basin; Table 2). A downstream abrupt increase in $\mathrm{Qm}$ takes place in sample S3 (70.4\%; Table 3). This is accompanied by the presence of small quantities of carbonate lithics (Table 3; Fig. 3a and b). This downstream change in the sand composition is related to the input of recycled material from sedimentary source rocks at the entrance into the Tajo Basin (Mesozoic and Tertiary sandstone and limestone and Quatemary clastics). Near the confluence of the Sorbe and Henares rivers (sample S1), Qm is reduced and proportions of the lithic fragments increase. This is a consequence of the homogenization processes, as also seen in the Cañamares and Bornova rivers. The low quantity of sedimentary lithic fragments makes their relative abundances unreliable (Fig. 3b). However, dolomitic fragments (Lsd) are poorly represented compared with sands from the Bornova and Cañamares rivers. $P / F$ values are especially low in this area reflecting the supply from Mesozoic arkoses (0.28 and $\mathbf{0 . 2 7}$ in samples $\mathrm{S} 1$ and $\mathrm{S} 3$, respectively).

The heavy mineral suites in the Sorbe River are diverse. Transparent non-micaceous species become enriched downstream, while opaques remain virtually unchanged throughout (samples S2 and S1, Table 4). Both upstream (S2) and downstream (S1) samples are dominated by staurolite and apatite. When the river enters the Tajo Basin (sample S1), characteristic sedimentary heavy minerals, mainly $\mathrm{Ca}-\mathrm{Mg}$ carbonate and baryte, increase

\section{Modal sand composition of the Henares River}

The headwaters of the Henares River are located in the Iberian Range, to the east of the study area. In this region, Mesozoic carbonate and sandstone outcrops generate recycled sediments with considerable amounts of carbonate lithic fragments and multicyclic quartz grains (Table 3; Arribas and Arribas, 1991; Arribas et al., 1998; Tortosa and Arribas, 1998). Before the confluence of the first tributary from the Central System (Cañamares River), the modal composition of the Henares River sands is consistent with its sedimentaclastic provenance, showing high $\mathbf{Q m}$ contents and a prevalence of carbonate lithic fragments over the total lithic fragments (sample H8; Figs. 2a, b, 3a and b).

In the QmFLt diagram (Fig. 2a), the composition of sands from the analysed sector of the Henares River seems to be very homogeneous, with no drastic changes in composition related to supply from the tributaries. This is because sedimentaclastic and 
metamorphiclastic sources form similar plots on a QmFLt plot: both types of supplies are feldspar-poor and may produce variable quantities of quartz and lithic fragments. The high content of quartz (63.7 \pm 4.6) and the dominance of lithic fragments over feldspars reflect their mixed metamorphi/sedimentaclastic provenance (quartzolithic sand-suites derived from recycled orogens; cf. Dickinson, 1985). Differences in sand composition are more evident when lithic grains contents are compared.

The entrance of the Cañamares River (sample $\mathrm{Cl}$ ) into the Henares River produces an increase in $\mathrm{F}$ content (sample H7; Fig. 2a). The corresponding dilution of sedimentary lithic fragments from $93.5 \%$ (sample H8) to $67.2 \%$ (sample H7) is caused by the major supply of metamorphic lithics from the Cañamares River (Fig. 2b and 3a; Table 2). In addition, the presence of volcanic lithic fragments in the mixed sand (sample H7) is consistent with the contribution from the Cañamares River. The mixing process between sample $\mathrm{H} 8$ and sample $\mathrm{Cl}$ to produce the $\mathrm{H} 7$ sample is clearly evident on the various ternary diagrams QmFLt, LmLvLs and LmLssLsc (Figs. 2a, b and $3 \mathrm{a}$ ), with sample $\mathrm{H} 7$ clearly falling on the joining line between samples $\mathrm{H} 8$ and $\mathrm{Cl}$. Further, the position of $\mathrm{H7}$ on this line is essentially equidistant between samples $\mathrm{H} 8$ and $\mathrm{Cl}$. Thus, mixing equal amounts of Henares and Cañamares River sand appears to explain the composition of the $\mathrm{H} 7$ sample.

Qm contents in the Henares River increase after the confluence with the Bomova River (sample H5 compared with samples H6 and H7). This increase can not be related to a mixing process with the Bornova River, because both B1 and H6 sand samples have lower $\mathbf{Q m}$ contents than sample H5 (Fig. 2a). In this area, river terraces with a quartzose composition are well developed (Portero et al., 1991), and thus, the increase in $\mathbf{Q m}$ particles may be related to reworking of Henares River terrace deposits. Supply of metamorphic lithic fragments from the Bornova River produces a dilution of sedimentary lithic fragments in the Henares River sands and the loss of volcanic lithic fragments previously acquired from the Cañamares River (sample H5; Figs. 2b and 3a).

Between the confluence of the Bornova and Sorbe rivers, the course of the Henares River is $25 \mathrm{~km}$ long. This $25 \mathrm{~km}$ is influenced by small seasonal, intermittent tributaries with limited drainage basins flowing mainly across the Tajo Basin sediments (Fig. 1C). However, there are minor outcrops of slate and Mesozoic carbonate source rocks in their headwaters. These outcrops play an important role, providing sedimentary and metamorphic lithic fragments to the Henares sediments (samples H4, H3 and H2; Figs. 2a and 3a). In total, the input from these local ributaries causes a decrease in Qm particles of nearly 10\% even though the Henares River reworks its own deposits and Tertiary clastics from the Tajo Basin infill.

The confluence of the Sorbe River causes a further decrease in Qm (sample H1; Fig. 2a) due to the supply of detritus with greater quantities of lithic fragments. However, the Sorbe River has little influence on the lithic fragment population of Henares River sand, which has similar proportions of lithic types before and after the confluences (samples $\mathrm{H} 2$ and H1; Figs. $2 \mathrm{~b}$ and $3 \mathrm{~b}$ ). As noted with the Cañamares River, the change in composition can be modelled by two component mixing of samples $\mathrm{H} 2$ and $\mathrm{S} 1$. In the QmFLt diagram, the resulting sand $(\mathrm{Hl})$ plots approximately halfway along the line joining the two end-members. However, on LmLvLs, LmLssLsc and LssLsdLsl diagrams (Figs. 2b, 3a and b), the mixed sand plots close to the Henares end-member. Thus, the Henares end-member exerts a greater influence on the composition of resultant sand than the Sorbe end-member. Therefore, the contribution made by the Sorbe River causes little change in the lithic population of the Henares River.

Heavy mineral assemblages along the Henares River course are diverse than those of its tributaries (Table 4). The heavy mineral assemblage of sample H8 is considered to be representative of the Iberian Range, before any supply from the Central System. The Cañamares catchment (sample C2) supplies an assemblage very similar to that observed in sample H8. Thus, no significant change occurs between the heavy mineral suites in sample $\mathrm{H} 8$ and $\mathrm{H} 7$ (after the entrance of Cañamares River), both assemblages being dominated by $\mathrm{Ca}-\mathrm{Mg}$ carbonate $(17.3 \%$; sample $\mathrm{H} 8)$ or staurolite $(11.5 \%$; sample $\mathrm{H} 7)$. However, there are significantly fewer Ca-I.Ig carbonate grains in $\mathrm{H} 7(1.8 \%)$ compared with $\mathrm{H} 8$ (17.3\%).

One of the most marked downstream variations along the Henares course, is the increase in garnet in H5 (23.6\%). This can be related to the widespread outcrop of gneissic source rocks in the Bomova 
drainage basin (sample B1; Table 4). In H5, relatively high frequencies are also shown by andalusite (6.3\%), tourmaline (4.1\%) and staurolite $(3.7 \%)$. The decrease in staurolite, compared with previous Henares samples ( $\mathrm{H} 8$ and H7) is explained by the mixing with low-staurolite heavy mineral suites from the Bornova River (samples B1 and B2). Ca-amphibole (green hornblende) is virtually absent in the upstream Henares sand (sample H8), but reaches a maximum in sample H5 (1.3\%). The most downstream sample (H1) is dominated by staurolite, zircon and apatite (Table 4). The heavy mineral assemblage in $\mathrm{Hl}$ is considerably influenced by the Sorbe River (S1) conribution, with the increase of staurolite and $\mathrm{Ca}-$ $\mathrm{Mg}$ carbonate, and the decrease of andalusite, gamet and tourmaline, being readily explained by this mixing process.

\section{Concluding discussion}

\subsection{The mixing source provenance}

The modal composition of sands from the Henares River and their ributaries (Cañamares, Bomova and Sorbe) is consistent with derivation from a mixture of metamorphic and sedimentary source rocks. This is manifested by generally low feldspar content with variable amounts of quartz and lithic fragments. Near all analysed samples plot in a restricted area on a $\mathrm{QmFLt}$ diagram (Fig. 2a) with high $\mathrm{Qm}$ contents (50-70\% approx.) and a general dominance of lithic fragments over feldspar. This modal composition is considered as quartzolithic (e.g. Dickinson, 1985). Within this cluster, the Cañamares and Bornova samples (except B3) plot closer to the feldspar pole, resulting from erosion of feldspathic gneiss that crops out in these drainage basins (Fig. 2a). Plots of Henares River sands in the QmFLt diagram show no drastic changes associated with the tributary supplies, maintaining a nearly constant composition along the fluvial course.

The Henares River receives its supply on the scale of a mountain range (Group II of Ingersoll et al., 1993). Several authors have found similar compositional modes in equivalent geological settings characterized by a wide variety of bedrock lithologies (e.g. Mack, 1981; Girty and Armitage, 1989; Critelli and
Le Pera, 1999). Data from the Holocene Colorado River sands (Girty and Armitage, 1989) are particularly similar in terms of the QmFLt diagram. In both cases, climate (arid to semiarid) acts to preserve carbonate lithic grains (Lsc). Similar preservation has been observed by other authors in sands and sandstones of sedimentaclastic origin (e.g. Ingersoll et al., 1987; Díaz-Molina and Tortosa, 1996; Arribas et al., 1998; Tortosa and Arribas, 1998). By contrast, in humid climates depletion or even complete disappearance of carbonate lithics has been reported Mack, 1981). There are exceptions to this general rule, such as the Lao River (northern Tyrrhenian coast of Calabria) where sands are composed mainly of carbonate extrabasinal grains (calclithite) even though the climate is humid to subhumid (Le Pera and Critelli, 1997). In this case, the very rough topography of the Lao drainage basin plays a decisive role, causing rapid transport of detritus, which minimised the effects of climate. In northern Calabria, modem sand petrofacies provide good examples of mixed-source sediments (e.g. Le Pera and Critelli, 1997; Le Pera, 1998). In this area, sands from mixed metamorphic and sedimentary sources have lower $\mathrm{Qm}$ contents than in sands from the Henares and its ributaries. We consider that this is due to the lack of siliciclastic formations in the Calabrian sedimentary bedrock, highlighting the relevance of this type of sedimentary rock as a major quartz grain producer.

The results of mixing main channel sands with tributary inputs can be analysed in the QmFLt diagram (Fig. 2a). Because all the lithic fragments are included in the Lt pole, this diagram does not discriminate between sedarenitic (calclithitic) and phyllarenitic sands. This is shown by samples S2 and B3, which have very different lithic types (metamorphic and sedimentary lithics, respectively) but plot very close together (Fig. 2a). Thus, this diagram is not useful in interpreting mixed metamorphic and sedimentary lithic sands. More suitable ternary diagrams to discriminate between these sources include diagrams using several types of lithic fragments (LmLvLs, LmLssLsc and LssLsdLsl; Figs. 2b and 3 ).

The heavy mineral suite along the Henares River varies downstream, with variations resulting from input from source rocks of the Central System. There is a detectable difference between the heavy 

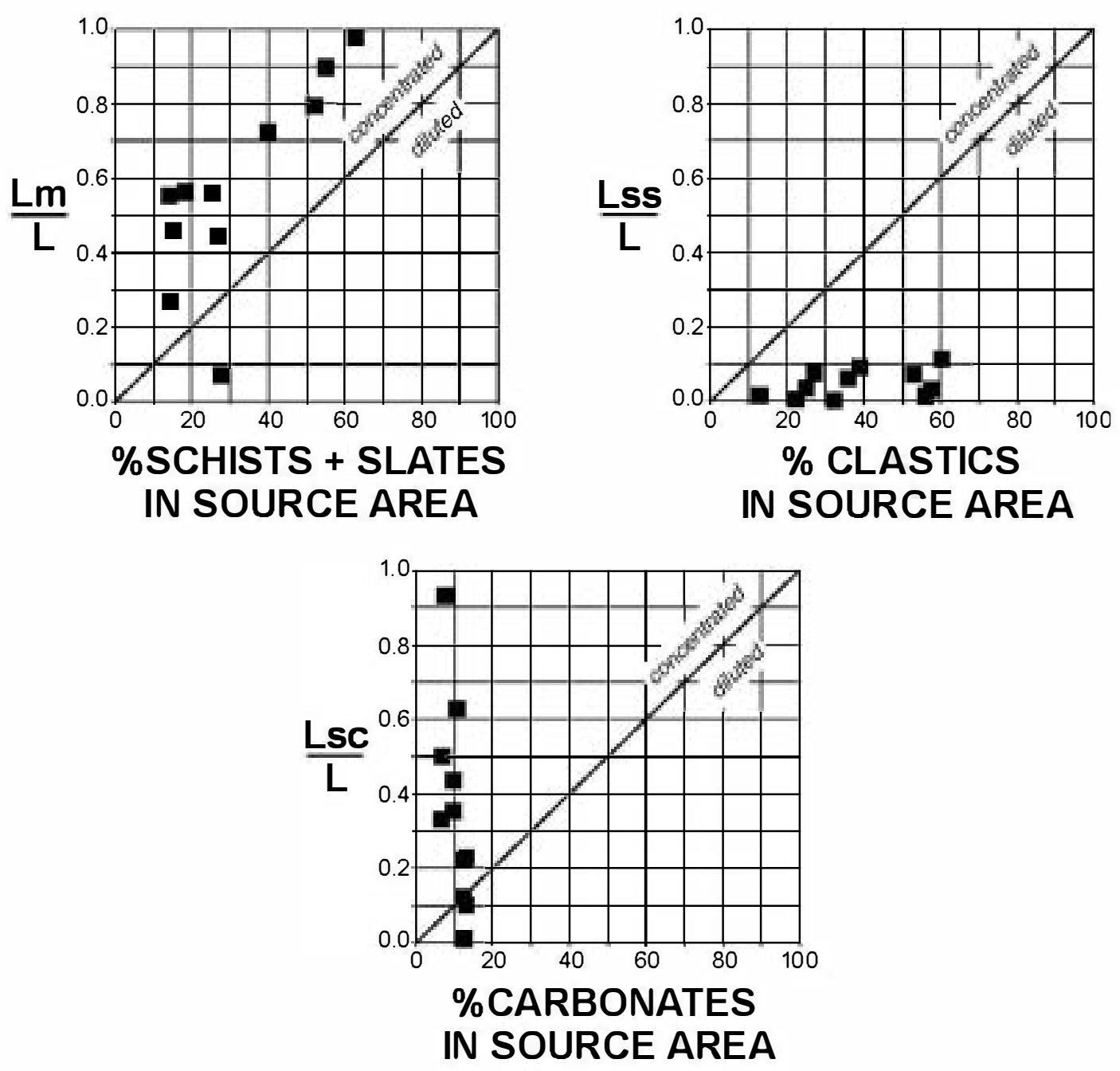

Fig. 4. Plots of several petrographic indices concerning lithic fragments $(\mathrm{L})$ in sands versus the percent of outcrop area of their bedrock. Lm: metamorphic lithic fragments. Lss: sedimentary siliciclastic lithic fragments. Lsc: sedimentary carbonate lithic fragments. L: total lithic fragments.

mineral suite in the upstream sands recycled from the Iberian Range (H8) and those from the Central System (H7-Hl) (Table 4). Dilution results in a downstream reduction of $\mathrm{Ca}-\mathrm{Mg}$ carbonate and $\mathrm{Fe}$-carbonate grains, as a result of addition of mixed sedimentary-metamorphic sources from the incoming tributaries. This supports the evidence for mixing processes shown by lithic grain trends, resulting in significant dilution of Henares detritus by the introduction of sand supplied by the tributaries.

\subsection{Contrast between source and sand composition}

Sediment composition during initial stages of sand generation is mostly controlled by the capability of a specific lithology to produce sand (e.g. McBride and Picard, 1987; Heins, 1993; Palomares and Arribas, 1993). Quantitative analysis of mixing relationships between two different source rocks depends on whether both rock types generate clear diagnostic detrital grains (Molinaroli and Basu, 1993). As discussed previously, sands from the Cañamares, 


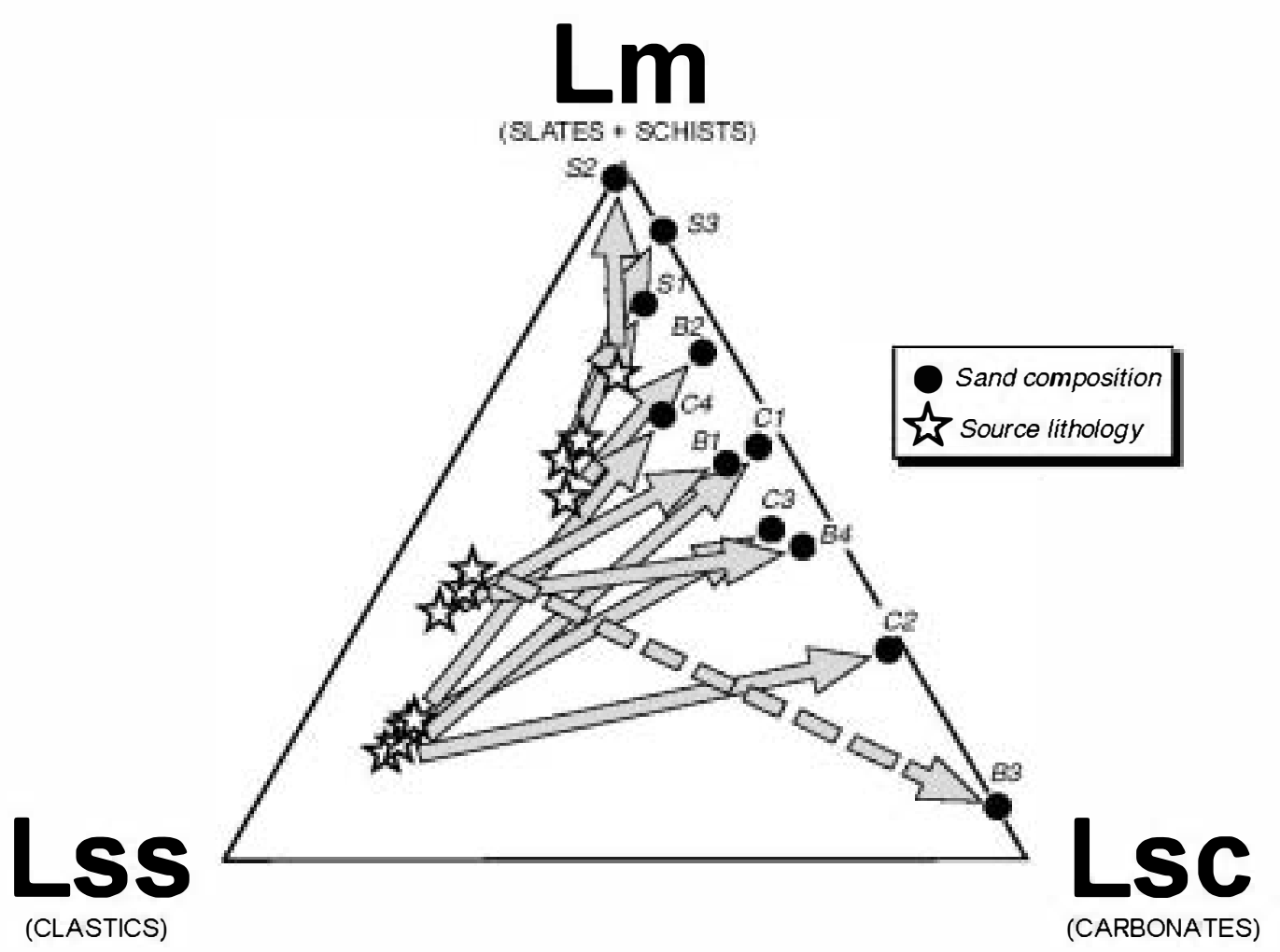

Fig. 5. Ternary diagram show ing the relative proportion of lithic grains (LmLssLsc) in sands from the Cañamares, Bornova and Sorbe rivers (black circles) and the corresponding proportions of bedrock lithologies (slates plus schists, clastics, and carbonates) at the sub-basins (white stars). Arrows link source and sediment compositions.

Bornova and Sorbe rivers are good examples of mixed deposits from a variety of source rocks. The quantification of the abundance of the different source rock types in each drainage sub-basin (Table 2) permits comparison with petrographic data from the sands. Because lithic grains are unequivocally related to their source, the contrast between their abundance in the sand and the areal extent of the source lithology in the drainage basin, allows us to evaluate the extent to which each grain type is representative of that source. In Fig. 4 are represented perographic indices concerning specific lithic grains to total lithics versus the specific source rock ratio (Mack, 1981). Proportions of metamorphic lithic grains are directly related to the relative distribution of slate and schist in the source area. The percentage of metamorphic lithic grains to total lithic grains $(\mathrm{Lm} / \mathrm{L})$ exceeds the relative proportion of these lithologies in the source area. This means that metamorphic lithic grains tend to concentrate in the lithic grain population, overrepresenting the proportion of this lithology in the source. By contrast, siliciclastic lithic grains are under-represented, with very low values of Lss/L index. This may be the result of low production of lithic grains from sedimentary clastic sources, causing a great dilution of these grains in the lithic grain population. Finally, carbonate lithic fragments are more variable with respect to total lithics, even when this lithology is poorly represented in the source (less than 15\%). Local supplies from carbonate sources may produce important increases of Lsc in short channel tracts (McBride and Picard, 1987). In the Cañamares, Bornova and Sorbe rivers, Lsc grain contents rise as a result of the presence of local Mesozoic carbonate rocks near the limit between the Central System and the Tajo Basin.

A similar $\mathrm{Lm} / \mathrm{L}$ trend was observed by Mack (1981) under humid climate conditions, although 
this author found minor concen ration of Lm grains in L population. McBride and Picard (1987) found that shale fragments are under-represented in sands of Graveglia River (Liguria, Italy), under a humid Mediterranean climate. Thus, the high Lm concentration in the lithic population observed in the Henares tributaries may be related to the semi-arid conditions in the drainage basins. Lm proportions may be boosted by the depletion of Lss grains, due to the interrelation of these parameters (Fig. 4). Cavazza et al. (1993) outlined the fact that many sedimentary rocks are systematically overlooked as source materials because of their low preservation potential in the sand fraction during the sedimentary cycle.

To better understand the relationships between the three lithic grains considered (Lm, Lss and Lsc) and the proportions of the corresponding source lithologies, data from both sources and sands have been plotted together in a LmLssLsc ternary diagram (Fig. 5). This again manifests the poor representation of Lss in sands compared with source lithology, expressed by depletion of these grains from source to sand. This is due to the poor capability of siliciclastic sedimentary rocks to generate lithic components.

The presence of more than $50 \%$ of slate plus schist with respect to other sedimentary rocks at the source, guarantees more than $75 \%$ of $\mathrm{Lm}$ in the lithic population. Carbonate lithic grains concentrate in the sands when slate plus schist are less than $50 \%$ at the source. The abnormal concentration of Lsc in sample $\mathbf{B} 3$ has been explained by local supplies from Mesozoic carbonate formations

Palomares and Arribas (1993) introduced the concept of sand generation index (SGI) to evaluate the relative capacity of a source rock to generate sand. This index is calculated taking every type of sand grains into account, and as a consequence it is necessary to know the composition of sands from each source rock type involved. In the Henares case, the complexity of the lithologies in the compound sources and the uncertainty of the provenance of some grain types (such as quartz or feldspar) obstruct a quantification of the SGI for the different lithologies considered. However, a qualitative assessment can be carried out if we consider only the lithic population.

Interpretation of the heavy mineral distribution pattern of the sands revealed that the percent frequencies of the main species are source-sensitive (e.g. Morton and Johnsson, 1993). The accurate representation of source rock by the heavy mineral assemblages is interpreted as a result of the weathering-limited erosion regime, within the Iberian Range and Central System, which has not affected the diversity of the heavy mineral assemblages taken into the transport system. Here even such unstable minerals such as andalusite and amphibole are represented in the sands. This conclusion is in accord with earlier studies (e.g. Mange-Rajetzky, 1983; Morton and Smale, 1990; Ibbeken and Schleyer, 1991; Morton and Johnsson, 1993) which show that where the detritus has only a short residence time in soil-forming environment, there is little or no effect on mineral proportions and diversity.

\subsection{Slope and transport effects on sand composition}

Slope and transport are important variables controlling sand composition (Johnsson, 1993). Slope controls the duration of weathering during pedogenesis, reducing the exposure time of sediments to be weathered (i.e. Stallard, 1985). The uniformity of the slope values in the different rocks constituting the drainage basins of the Cañamares, Bomova and Sorbe rivers (Table 2) precludes an evaluation of the influence of slope on sand composition. However, there are differences in slopes between Mesozoic rocks cropping out at the north (gentle slopes and near horizontal dips) and at the south (steep slopes and dips) of the drainage basins (compare Fig. 1B and D). Sands collected at the heads of the three drainage basins ( $C 4,82$ and S2) have the greatest dilution of sedimentary lithic grains, manifested by the steepest arrows in Fig. 5. Conversely, the greatest concentration of carbonates in each drainage basin corresponds to samples collected downstream of the southern Mesozoic formations (C2, B3 and S3). In addition, the slope effect on sand composition may be influenced by abrasion during transport.

Along the course of the Henares River, sand compositions show no drastic variations in the QmFLt diagram (Fig. 2a). Variations in the contents of lithic grain types (Figs. 2b and 3a) correspond to supplies from its tributaries. Lm increases from $\mathrm{H} 8$ to $\mathrm{H} 5$, but decreases again from $\mathrm{H} 3$ to $\mathrm{H} 1$. Thus, we can conclude that abrasion during transport exerts no significant changes in sand composition along the 
Henares River. The fact that inputs from the Cañamares, Bornova and Sorbe do not produce a consistent or permanent change in composition on Henares sands is manifested by the loss of Lm during the last racts of the Henares course (Figs. 2b and 3a). This effect is probably related not only to maturation processes during transport, but also to mixing with previous terrace deposits of the Henares fluvial system. Thus, reworking plays a significant role in homogenisation of the composition of Henares sands as a second-order sampling group defined by Ingersoll et al. (1993).

\subsection{The recycling problem}

Signals of recycling of grains from sedimentary and metasedimentary bedrock are apparent in the composition of sands from the Henares River and its tributaries. Criteria for the identification of multicyclic detritus listed by Zuffa (1987) are verified here as useful. However, a quantitative evaluation of recycling is very complex. The main factors that make this difficult are: (1) the great diversity of source rocks that may produce a wide spectrum of sand composition, from deposits with 'common' grains (such as monocrystalline quartz generated from siliciclastic successions), to deposits consisting of specific diagnostic lithic grains (such as carbonate lithics); (2) the diverse potentiality of these bedrocks to generate sand grains (Arribas et al., 1998) possibly causing drastic dilution of some diagnostic grains (McBride and Picard, 1987); and (3) the low preservation potential of some sedimentary and metasedimentary lithic fragments (as carbonate lithics) during ransport because of their fragility (Cameron and Blatt, 1971; Graham et al., 1986) or to the influence of climatic conditions (Mack, 1981; Cavazza et al., 1993). To isolate all these factors a wider petrographic data base of these types of sediments in other geographic and climatic settings is needed.

\section{Acknowledgements}

This work was funded by a Bilateral Italian Consiglio Nazionale delle Ricerche (CNR)-Spanish Consejo Superior de Investigation Cientificas (CSIC) grant (Project: The effects of lithology, morphology, transport and depositional environment on quantitative provenance of modern sand (Spain,
Calabria): implications for geographic distribution of actualistic petrofacies; Resps. J. Arribas and S. Critelli). The paper is also a contribution to the spanish DGICYT project PB93-178. We thank G. Garzon for her help and suggestions in understanding Quaternary and recent deposits of the Henares River. We are grateful to W. Cavazza, K.A.W Crook, E.F. McBride and A.C. Morton for reviews and helpful discussions on an early version of the manuscript.

\section{Ref erences}

Arribas, Ma E., Díaz-Molina, M., López-Martínez, N., Portero, J.M., 1983. El abanico aluvial paleógeno de Beleña de Sorbe (Cuenca del Tajo): facies, relaciones espaciales y evolución. X Congreso Nacional de Sedimentología, Menorca, Comunicaciones, vol. 1, pp. 34-38.

Arribas, J., Arribas, Ma.E., 1991. Petrographic evidence of diff erent provenance in two alluvial fan systems (Palaeogene of the northem Tajo Basin, Spain). In: Morton, A.C., Todd, S.P., Haughton, P.D.W. (Eds.), Developments in Sedimentary Provenance Studies, Geol. Soc. Spec. Publ., vol. 57, pp. 263-271.

Arribas, J., de la Peña, J.A., Tortosa, A., 1998. Detrital modes in sedimentoclastic sands from first-order streams of the Iberian Range. Spain: The eff ect of Source Lithology. 15th International Sedimentology Congress, pp. 153-154.

Basu, A., 1976. Petrology of Holocene fluvial sand derived from plutonic source rocks. implications to paleoclimatic interpretations. J. Sediment. Petrol. 46, 694-709.

Basu, A., 1985. Reading provenance from detrital quartz. In: Zuff a, G.G. (Ed.), Provenance of Arenites, Reidel, Dordrecht, pp. 231 248 .

Cameron, K.L., Blatt, H., 1971. Durabilities of sand-size schist and "volcanic" rock fragments during fluvial transport, Elk Creek, Black Hills, South Dakota. J. Sediment. Petrol. 41, 565-576.

Capote, R., Casquet, C., Fernandez-Casals, M.J., 1982.L os grandes complejos estructurales del Sistema Central: Modelo de evolución tectonometamórfica. Revista de la Real Academia de Ciencias Exactas Físicas y Naturales 76, 313-331

Carson, M.A., Kirkby, M.J., 1972. Hillslope Form and Process, Cambridge University Press, Cambridge, $475 \mathrm{pp}$.

Casquet, C., Fernández-Casals, M.J., 1981. Las anfibolitas de la región de Buitrago de Lozoya (Sistema Central Español). Cuademos de Geología Ibérica 7, 121-134

Cavazza, W., Zuffa, G.G., Camporesi, C., Ferretti, C., 1993. Sedimentary recycling in a temperate climate drainage basin (Senio River, north-central Italy): composition of soil profiles and fluvial deposits. In: Johnsson, M.J., Basu, A. (Eds.), Processes Controlling the Composition of Clastic Sediments, Geol. Soc. Am. Special Paper, vol. 284, pp. 247-261.

Critelli, S., Ingersoll, R.V., 1995. Interpretation of neovolcanic versus palaeovolcanic sand grains: an example from Miocene 
deep-marine sandstone of the Topanga Group (Southem California). Sedimentology 42, 783-804.

Critelli, S., Le Pera, E., 1994. Detrital modes and provenance of Miocenesandstonesand Modem sands of the Southem Apennines thrust-top basins (Italy). J. Sediment. Res. A64, 824-835.

Critelli, S., Le Pera, E., Ingersoll, R.V., 1997. The effects of source lithology, transport, deposition and sampling scale on the composition of southern California sand. Sedimentology 44 , $653-671$

Critelli, S., Le Pera, E., 1999. Provenance relations and modem sand petrofacies in an uplifted thrust-belt, northern Calabria, Italy. In: A. Basu, R. Valloni (Eds.), Quantitative Provenance Studies in Italy. Memorie Descrittive della Carta Geologica D'Italia, Special Issue (in press).

DeCelles, P.G., Hertel, F., 1989. Petrology offluvial sands from the Amazonian foreland basin, Peru and Bolivia. Geol. Soc. Am. Bull. 101, 1552-1562.

Díaz-Molina, M., Tortosa, A., 1996. Fluvial fans of the Loranca Basin, Late Oligocene-Early Miocene, central Spain. In: Friend, P.F., Dabrio, C.J. (Eds.), Tertiary Basins of Spain: The Stratigraphic Record of Crustal Kinematics, World and Regional Geology, vol. 6. Cambridge University Press, Cambridge, pp. 300-307.

Dickinson, W.R., 1985. Interpreting provenance relations from detrital modes of sandstones. In: Zuffa, G.G. (Ed.), Provenance of Arenites, Reidel, Dordrecht, pp. 333-361.

Dickinson, W.R., 1988. Provenance and sediment dispersal in relation to paleotectonics and paleogeography of sedimentary basins. In: Kleinspehn, K.L., Paola, C. (Eds.), New Perspectives in Basin Analysis. Frontiers in Sedimentary Geology, Springer, New York, pp. 3-25.

Girty, G.H., Amitage, A., 1989. Composition of Holocene Colorado River sand: an example of mix ed-provenance sand derived from multiple tectonic elements of the cordilleran continental margin. J. Sediment. Petrol. 59, 597-604.

Graham, S.A., Tolson, R.B., Decelles, P.G., Ingersoll, R.V., Bargar, E., Caldwell, M., Cavazza, W., Edwards, D.P., Follo, M.F., Handschy, M.F., Lemke, J.F., Moxton, I., Rice, R., Smith, G.A., White, J., 1986. Provenance modelling as a technique for analysing source terrane evolution and controls on foreland sedimentation. In: Allen, P.A., Homew ood, P. (Eds.), Foreland Basins. Special Publication of the International Association of Sedimentologists, vol. 8, pp. 425-436

Grantham, J.H., Velbel, M.A., 1988. The influence of climate and topography on rock-fragment abundance in modem fluvial sands of the Southem Blue Ridge Mountains, North Carolina. J. Sediment. Petrol. 58, 219-227.

Heins, W.A., 1993. Source rock texture versus climate and topography controls on the composition of modem, plutoniclastic sand. In: Johnsson, M.J., Basu, A. (Eds.), Processes Controlling the Composition of Clastic Sediments, Geol. Soc. Am. Special Paper, vol. 284, pp. 135-146.

Ibbeken, H., Schleyer, R., 1991. Source and Sediment. A Case Study of Provenance and Mass Balance at an Active Plate Margin (Calabria, Southern Italy), Springer, Berlin, $286 \mathrm{pp}$

I.G.N., 1991. Atlas Nacional de España. Sección II, Grupo 9, 'Climatología', 9.24pp.
Ingersoll, R.V., Bullard, T.F., Ford, R.L., Grimm, J.P., Pickle, J.D., Sares, S.W., 1984. The effect of grain size on detrital modes. a test of the Gazzi-Dickinson point-counting method. J. Sediment. Petrol. 54, 103-116.

Ingersoll, R.V., Cavazza, W., Graham, S.A., 1987. Provenance of impure calclithites in the Laramide foreland of southwestern Montana (Indiana University Graduate Field Seminar Participants). J. Sediment. Petrol. 57, 995-1003.

Ingersoll, R.V., Kretchmer, A.G., V alles, P.K., 1993. The eff ect of sampling scale on actualistic sandstone petrofacies. Sedimentology 40, 937-953.

Johnsson, M.J., 1993. The system controlling the composition of clastic sediments. In: Johnsson, M.J., Basu, A. (Eds.), Processes Controlling the Composition of Clastic Sediments, Geol. Soc. Am. Special Paper, vol. 284, pp. 1-19.

Johnsson, M.J., Stallard, R.F., 1989. Physiographic controls of the composition of fluvial sands derived from volcanic and sedimentary terrains on Barro Colorado Island, Panama. J. Sediment. Petrol. 59, 768-781.

Le Pera, E., Critelli, S., 1997. Sourceland controls on the composition of beach and fluvial sand of the northem Tyrrhenian coast of Calabria, Italy: implications for actualistic petrofacies. Sediment. Geol. 110, 81-97.

Le Pera, E., 1998. Relazioni composizionali tra aree fonte e sabbie fluviali, costiere e marine attuali e recenti in Calabria settentrionale. Unpublished PhD Thesis, University of Bologna, 278 pp.

López-Ruiz, J., Aparicio, A., García Cacho, L., 1975. El metamorfismo de la Sierra del Guadarrama, Sistema Central español. Memorias del Instituto Geológico y Minero de España 86, 127.

Mack, G.H., 1981. Composition of modem stream sand in a humid climate derived from a low-grade metamorphic and sedimentary foreland fold-thrust belt of north Georgia. J. Sediment. Petrol. 51, 1247-1258.

Mange-Rajetzky, M.A., 1983. Sediment dispersal from source to shelf on an active continental margin, S. Turkey. Marine Geol. 52, 1-26.

Marsaglia, K.M., 1993. Basaltic island sand provenance. In: Johnsson, M.J., Basu, A. (Eds.), Processes Controlling the Composition of Clastic Sediments, Geol. Soc. Am. Special Paper, vol. 284 , pp. $41-65$.

McBride, E.F., Picard, M.D., 1987. Downstream changes in sand composition, roundness, and gravel size in a short-headed highgradient stream, northwestern Italy. J. Sediment. Petrol. 57, $1018-1026$.

Molinaroli, E., Basu, A., 1993. Toward quantitative provenance analysis A brief review and case study. In: Johnsson, M.J., Basu, A. (Eds.), Processes Controlling the Composition of Clastic Sediments, Geol. Soc. Am. Special Paper, vol. 284, pp. 323-333.

Montesinos, S., Arribas, J., 1998. Source area versus detrital products: A geographical information system approach. 15th International Sedimentological Congress, pp. 558-559.

Morton, A.C., Smale, D., 1990. The effect of transport and weathering on heavy minerals from the Cascade River, New Zealand. Sedimentary Geol. 68, 117-123.

Morton, A.C., Johnsson, M.J., 1993. Factors influencing the composition of detrital heavy mineral suites in Holocene sands of the 
Apure River drainage basin, Venezuela. In: Johnsson, M.J., Basu, A. (Eds.), Processes Controlling the Composition of Clastic Sediments, Geol. Soc. Am. Special Paper, vol. 284, pp. $171-185$.

Navidad, M., 1978. Las series glandulares "Ollo de Sapo" en los sectores nordoccidental y centro-oriental del macizo Ibérico. Estudios Geológicos 34, 511-528.

Nesbitt, H.W., Young, G.M., McLennan, S.M., Keays, R.R., 1996. Effects of chemical weathering and sorting on the petrogenesis of siliciclastic sediments, with implications for provenance studies. J. Geol. 104, 525-542.

Palomares, M., Arribas, J., 1993. Modem stream sands from compound crystalline sources. Composition and sand generation index. In: Johnsson, M.J., Basu, A. (Eds.), Processes Controlling the Composition of Clastic Sediments, Geol. Soc. Am. Special Paper, vol. 284, pp. 313-322.

Parfenoff, A., Pomerol, C., Tourenq, J., 1970. Les minéraux en grains. Méthodes d'étude et détermination. Paris, Masson et Cie, Ed., 578pp.

Portero, J.M., Aznar, J.M., Diaz Molina, M., Pérez Gonzalez, A., Gallardo, J., Gonzalez Lodeiro, F., Aguilar, M.J., Leal, M.C., 1991. Mapa Geológico de España E. 1:50.000, Hoja n` 486 (Jadraque). Memoria explicativa. Inst. Geol. Min. España, 77pp.

Potter, P.E., 1986. South America and a few grains of sand Part I Beach sands. J. Geol. 94, 301-319.

Stallard, R.F., 1985. River chemistry, geology, geomorphology, and soils in the Amazon and Orinoco basins. In: Drever, J.I. (Ed.), The Chemistry of Weathering, Reidel, Dordrecht, pp. 293-316.
Suttner, L.J., 1974. Sedimentary petrographic provinces: an evaluation. In: Ross, C.A. (Ed.), Paleogeographic Provinces and Provinciality, Soc. Econ. Paleontol. Mineral. Special Publication, vol. 21, pp. 75-84

Tortosa, A., Arribas, J., Garzón, G., Fernández, P., Palomares, M., 1997. Análisis petrológico de los depósitos de terrazas aplicado al estudio de los procesos de captura en los ríos Adaja, V oltoyay Eresma (Províncias de Segovia y Valladolid). Rev. Soc. Geol. España 10 (1-2), $131-145$.

Tortosa, A., Arribas, J., 1998. Evolution of sandstone composition in a continental foreland basin. Loranca Basin, Spain. 15th International Sedimentological congress, pp. 769-770

Valloni, R., 1985. Reading provenance from modem marine sands In: Zuffa, G.G. (Ed.), Provenance of Arenites, Reidel, Dordrecht, pp. 309-332.

Vialette, Y., Casquet, C., Fuster, J.M., Ibarrola, E., Navidad, M., Peinado, M., Villaseca, C., 1986. Orogenic granitic magmatism of prehercynian age. Terra Cognita ICOG 6 meeting 6, 1602

Wilson, L., 1969. Les rélations entre les processus géomorphologiques et le cl imat moderne comme méthod de palé ocl imatologie. Rev. Géogr. Phis. Géol. Dinam. 11, 303-314.

Zuffa, G.G., 1985. Optical analyses of arenites: influence of methodology on compositional results. In: Zuffa, G.G. (Ed.), Provenance of Arenites, Reidel, Dordrecht, pp. 165-189.

Zuffa, G.G., 1987. Unravelling hinterland and offshore paleogeography from deep-w ater arenites. In: Leggett, J.K., Zuffa, G.G. (Eds.), Deep-Marine Clastic Sedimentology. Concepts and Case Studies, Graham and Trotman, London, pp. 39-61. 